\title{
Influences of Domain Symmetry on Supersonic Combustion Modeling
}

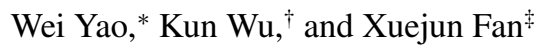 \\ Chinese Academy of Sciences, 100190 Beijing, People's Republic of China
}

DOI: $10.2514 / 1 . B 37227$

\begin{abstract}
High-fidelity Improved Delayed Detached Eddy Simulation modeling is applied to examine the influence of the symmetry plane on the supersonic flow and combustion characteristics in a round-to-elliptic-transition scramjet combustor. A quarterly split domain and the full domain are modeled by using up to 0.14 billion cells and a third-order scale-selective discretization scheme. The combustion chemistry is described by the finite-rate partially stirred reactor model based on multicomponent diffusion and a skeletal kerosene mechanism with 19 species/ 53 reactions. Time-averaged pressure, temperature, Mach number, and heat release rate are compared to examine the influence of the symmetry assumption on the mean flow fields. The exchanges of mass, momentum, and energy across the symmetry planes are analyzed. Considerable increases in the combustion efficiency and total pressure loss occur when applying the full domain, although with the similar mixing efficiency. Turbulence spectrum analysis confirms the $-8 / 3$ power-law for supersonic flows, and the inertial subrange is found to be shorter in the quarterdomain modeling. A comparison of the Borghi's diagrams shows that the data points shift from the flamelet mode to the thin reaction zone mode when using the full domain, and at least $1 / 3$ of them cannot be described by fast-chemistry combustion models.
\end{abstract}

$\begin{aligned} & \text { Nomenclature } \\ = & \text { cross-sectional area } \\ = & \text { specific heat, } \mathrm{J} / \mathrm{kg} \cdot \mathrm{K}) \\ = & \text { molar concentration of species } \alpha, \mathrm{mol} / \mathrm{m}^{3} \\ = & \text { model constant, } 0.09 \\ = & \text { mass and thermal diffusivity, } \mathrm{m}^{2} / \mathrm{s} \\ = & \text { Damköhler number } \\ = & \text { length scale in IDDES, } \mathrm{m} \\ = & \text { heat release rate, } \mathrm{W} \\ = & \text { absolute and total absolute enthalpy, } \mathrm{J} / \mathrm{kg} \\ = & \text { step height, } \mathrm{m} \\ = & \text { the forward and reverse rate constants of the } \\ = & \quad \text { th-elementary reaction } \\ = & \text { motions, } \mathrm{m}^{2} / \mathrm{s}^{2} \\ = & \text { unresolved turbulent kinetic energy, } \mathrm{m}^{2} / \mathrm{s}^{2} \\ = & \text { numbers of species, reactions and mesh cell } \\ = & \text { thickness of flame front, } \mathrm{m} \\ = & \text { Kolmogorov length scale, } \mathrm{m} \\ = & \text { thickness of preheating zone, } \mathrm{m} \\ = & \text { thickness of chemical reaction zone, } \mathrm{m} \\ = & \text { Mach number } \\ = & \text { mass flow rates, kg } / \mathrm{s} \\ = & \text { turbulent Prandtl number } \\ = & \text { static and total pressure, Pa }\end{aligned}$

Received 26 May 2018; revision received 15 November 2018; accepted for publication 15 November 2018; published online 28 December 2018. Copyright $\odot 2018$ by the American Institute of Aeronautics and Astronautics, Inc. All rights reserved. All requests for copying and permission to reprint should be submitted to CCC at www.copyright.com; employ the ISSN 0748-4658 (print) or 1533-3876 (online) to initiate your request. See also AIAA Rights and Permissions www.aiaa.org/randp

*Associate Professor, Key Laboratory of High Temperature Gas Dynamics, Institute of Mechanics; also School of Engineering Science, University of Chinese Academy of Sciences, 100049 Beijing, People's Republic of China; weiyao@imech.ac.cn. Member AIAA.

${ }^{\dagger}$ Ph.D. Student, Key Laboratory of High Temperature Gas Dynamics, Institute of Mechanics; also School of Engineering Science, University of Chinese Academy of Sciences, 100049 Beijing, People's Republic of China; wukun@imech.ac.cn.

Professor, Key Laboratory of High Temperature Gas Dynamics, Institute of Mechanics; also School of Engineering Science, University of Chinese Academy of Sciences, 100049 Beijing, People's Republic of China; xfan@imech.ac.cn. Lifetime Member AIAA (Corresponding Author).

$R, R_{u}$
$S_{i j}$
$S c_{t}$
$T, T_{t}$
$V, u_{i}, u_{i}^{\prime}$
$W$
$W_{0}$
$x$
$Y$
$y^{+}$
$\epsilon$
$\varepsilon$
$\eta_{\text {comb }}$
$\eta_{\text {mix }}$
$\eta_{P t}$
$\nu, \nu_{t}, \nu_{\text {eff }}$
$\nu_{\mathrm{CO}}$
$\xi_{\mathrm{st}}$
$\rho$
$\tilde{\tau}_{i j}, \tau_{i j}$
$\tau_{c}, \tau_{\delta}$
$\tau_{\text {mix }}, \tau_{\text {mix }, t}, \tau_{\text {mix }, l}$
$\tau_{0}, \tau_{t}, \tau_{\Delta}, \tau_{k}$
$\Phi$
$\tilde{\chi}$
$\Psi_{T, j}$
$\Psi_{\alpha, j}$
$\omega_{\alpha}$

$=$ gas constant $(\mathrm{J} /(\mathrm{kg} \cdot \mathrm{K}))$ and universal gas constant $(\approx 8.314 \mathrm{~J} /(\mathrm{mol} \cdot \mathrm{K}))$

$=$ strain rate tensor, $\mathrm{m} / \mathrm{s}$

$=$ turbulent Schmidt number

$=$ static and total temperature, $\mathrm{K}$

$=$ velocity vector, $i$ th-component and fluctuation, $\mathrm{m} / \mathrm{s}$

$=$ molecular weight, $\mathrm{g} / \mathrm{mol}$

$=$ channel width, $\mathrm{m}$

$=$ Cartesian coordinate in the $x$ direction

$=$ mass fraction

$=$ nondimensional wall distance

$=$ turbulence dissipation rate, $\mathrm{J} /(\mathrm{kg} \cdot \mathrm{s})$

$=$ small quantity to avoid zero division, usually $1 \times 10^{-30}$

$=$ combustion efficiency

$=$ mixing efficiency

$=$ total pressure loss

$=$ kinematic, turbulent, and effective viscosity, $\mathrm{m}^{2} / \mathrm{s}$

$=$ stoichiometric coefficient of $\mathrm{CO}_{2}$

$=$ stoichiometric mixture fraction

$=$ density, $\mathrm{kg} / \mathrm{m}^{3}$

$=$ viscous and Reynolds stress tensor, $\mathrm{kg} /\left(\mathrm{m} \cdot \mathrm{s}^{2}\right)$

$=$ time scales of flame front and chemical reaction zone, $\mathrm{s}$

$=$ micromixing time scale, its laminar and turbulent parts, $\mathrm{s}$

$=$ integral, SGS, Taylor and Kolmogorov time scales, $\mathrm{s}$

$=$ global equivalence ratio

$=$ mean scalar dissipation rate, $\mathrm{s}^{-1}$

$=$ turbulent enthalpy flux, $\mathrm{W} / \mathrm{m}^{2}$

$=$ turbulent species diffusion, $\mathrm{kg} /\left(\mathrm{m}^{2} \cdot \mathrm{s}\right)$

$=$ mass production of species $\alpha, \mathrm{kg} /\left(\mathrm{m}^{3} \cdot \mathrm{K}\right)$

Superscripts

$\sim$

$=$

$=$

Favre-averaged quantity

averaged quantity

\section{Subscripts}

air

$=$ quantity of the air crossflow 


$\begin{array}{ll}\mathrm{BL} & =\text { quantity of the boundary layer } \\ \text { fuel } & =\text { fuel quantity } \\ i, j & =\text { direction indices } \\ x & =\text { quantity at the streamwise location of } x \\ \alpha & =\end{array}$

\section{Introduction}

$\mathbf{S}$ YMMETRY boundary treatment has been frequently used in the large-eddy simulation (LES) modeling of symmetric and axisymmetric scramjet combustors [1-7], mainly to alleviate the huge computational cost. Moreover, from the presented results, the flow variables (e.g., wall pressure) seem to be well predicted. Such symmetry boundary treatment is generally acceptable for Reynolds-averaged Navier-Stokes (RANS) modelings because the time-averaged flow fields are supposed to be symmetric. However, it is questionable to apply the symmetry boundary condition for LES modelings, because unsteady flows would not obey any kinds of instantaneous symmetry restriction that would only emerge after time averaging. Theoretically, if the flow on the symmetric boundary is mainly supersonic and can be classified as the hyperbolic problem, the symmetry boundary condition can exert influence only on a restricted downstream region. For reacting flows, the influence of symmetry boundary may be more noticeable, because even a small exchange of high-temperature combustion gas through the symmetry plane may significantly affect the reactivity on both sides. It would be of practical engineering meaning to quantify the errors induced by using such symmetry boundary treatment in supersonic combustion modelings.

The flow symmetry in reacting systems is especially vulnerable and can be easily broken by changes in the boundary conditions (e.g., the area-expansion ratio [8], domain width and lateral boundary condition [9]) or even the flow properties (e.g., Lewis number [10]). The initial symmetry breaking bifurcation can be restricted artificially by the symmetry boundary through coercing the flux across the symmetry plane to zero, which will suppress coherent structures in the sizes larger than the split domain and provide an additional effect to stabilize the unsteady flow. For a full symmetric or axisymmetric domain modeling, even the time-averaged mass and energy exchanges across the symmetry plane are statistically conserving, and the instantaneous mass and energy exchanges may still stir the flow and strengthen the turbulence on both sides, which implies that the symmetry boundary treatment may suppress the combined entropy increase on the two sides. For reacting flows, the flow can become markedly asymmetric because of the asymmetric heat release on the two sides due to the exchanges of ignition energy and active radicals. Combustion provides a unique mechanism to deploy kinetic energy from the smallest scales to the system scales through the pressure-dilatation work [11] as well as the thermalexpansion-driven backscatter [12]. Because combustion reactions on the molecular level are inherently uncontrollable, the well-known butterfly effect in chaos theory suggests that a very asymmetric statistically averaged flow can be incubated from an initial symmetric flow. In practice, asymmetric burning has been frequently observed for symmetric or asymmetric combustors in the form of localized overheating or overpressurization, which may finally lead to serious engine accidents $[13,14]$. For axisymmetric flow configurations, swirling flows or azimuthal oscillations can spontaneously form and have been reproduced numerically $[8,10,15,16]$.

In this study, a kerosene-fueled scramjet combustor working at a flight Mach number of 6.5 is modeled by Improved Delayed Detached Eddy Simulation (IDDES) [17] with a quarter domain and the full domain, respectively. Streamwise measurements of wall pressure are first used to validate the predictions. Time-averaged and instantaneous fields are then analyzed by the aid of numerous indices to quantify the influence of the symmetric treatment on the predictions of combustor performances and mixing/combustion characteristics. The study would be useful to provide some guidelines for the future scramjet combustor modelings, where the huge computational cost is the critical hindering factor in implementing full-domain modelings.

\section{Experimental and Numerical Details}

\section{A. Experimental Case}

Figure 1 shows the schematics of the round-to-elliptic shapetransition scramjet combustor, respectively, through the major and minor axes of the elliptic cross section (from now on referred to as major and minor symmetry planes). The scramjet combustors are composed of three sections: a $600-\mathrm{mm}$-long isolator section with a $0.7^{\circ}$ divergence angle, an 800-mm-long burner section, and a 600 -mm-long expander section with a $4^{\circ}$ divergence angle. The inlet diameters of the three sections are 125, 132, and $170 \mathrm{~mm}$, respectively, and the diameter of the expander outlet is $212 \mathrm{~mm}$. In the elliptic burner section, the shortest semi-minor axis of $50 \mathrm{~mm}$ and the longest semi-major axis of $115.5 \mathrm{~mm}$ are located on a cross section of $425 \mathrm{~mm}$ downstream of the burner inlet. There are two circumvented cavities assembled in tandem in the burner section for flame anchoring and autoignition. The upstream and downstream cavities, respectively, have a distance of 228 and $578 \mathrm{~mm}$ from the burner inlet to their leading edges. Both the cavities have a depth of $15 \mathrm{~mm}$ and a length-to-depth ratio of 7 and thus are classified as open cavities. The aft walls of the cavities are at an angle of $45^{\circ}$ relative to the bottom of the cavity. The fuel is injected at $56 \mathrm{~mm}$ upstream of the upstream cavity from six 2.5-mm-diam injection portholes, whose locations are shown in Fig. 1 . The central injector is through the minor symmetry plane, and its neighbors are on its two opposite sides with a circumferential distance of $45 \mathrm{~mm}$. There is no injector through the major plane. Wall pressure is measured by the Motorola MPX2200 pressure transducers with an interval of $50 \mathrm{~mm}$.

The experiments with configurations listed in Table $\underline{1}$ are conducted in a continuous-flow supersonic combustion test platform to simulate a flight Mach number of 6.5. The incoming vitiated air supplied by burning hydrogen in the air with oxygen replenishment has a raised total temperature of $1600 \mathrm{~K}$, a mass flow rate of $3.6 \mathrm{~kg} / \mathrm{s}$, and a Mach number of 3.0 at the isolator inlet. The compositions of the vitiated air are $\mathrm{N}_{2}$ in $60.5 \%$ mole fraction, $\mathrm{O}_{2}$ in $21.7 \%$, and $\mathrm{H}_{2} \mathrm{O}$ in $17.8 \%$. The global equivalence ratio is $\Phi=0.8$, which is equally distributed between the injectors. Supercritical China RP-3 kerosene preheated by an electric ceramic heater before the injection is used to enhance its mixing with the vitiated air crossflow. Although no active-cooling facility is applied for the combustor walls, no obvious melting damage to the wall surface was observed after the $27 \mathrm{~s}$ test, indicating that the wall temperature is below the melting point of $1300 \mathrm{~K}$.

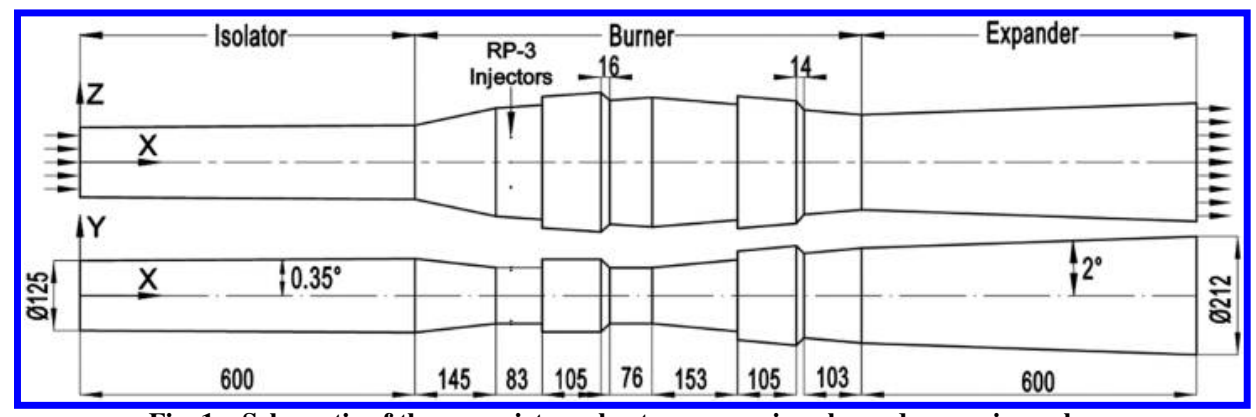

Fig. 1 Schematic of the scramjet combustor: up, major plane; down, minor plane. 
Table 1 Test case configurations

\begin{tabular}{lcccccccccc}
\hline \hline \multicolumn{1}{c}{ Vitiated air crossflow } & \multicolumn{4}{c}{ RP-3 jet } \\
\hline$P_{t, \text { air }}, \mathrm{MPa}$ & $T_{t, \text { air }}, \mathrm{K}$ & $\dot{m}_{\text {air }}, \mathrm{kg} / \mathrm{s}$ & $M a$ & $\mathrm{~N}_{2}, \mathrm{~mol} / \mathrm{mol}$ & $\mathrm{O}_{2}, \mathrm{~mol} / \mathrm{mol}$ & $\mathrm{H}_{2} \mathrm{O}, \mathrm{mol} / \mathrm{mol}$ & $P_{t, f}, \mathrm{MPa}$ & $T_{t, f} f T_{t, f}, \mathrm{~K}$ & $\dot{m}_{\text {fuel }}, \mathrm{kg} / \mathrm{s}$ & $\Phi$ \\
\hline 13.66 & 1581.7 & 3.5789 & 3.0 & $60.5 \%$ & $21.7 \%$ & $17.8 \%$ & 5.77 & 784 & 0.2181 & 0.8 \\
\hline \hline
\end{tabular}

\section{B. Flow Solver: AstroFoam}

The modelings are performed by the compressible reacting flow solver AstroFoam, which is developed from the standard compressible flow solver rhoCentralFoam [18] distributed with the finite-volume (FV) unstructured CFD package OpenFOAM V3.0.1 [19]. The main development is the adding of a full species transport module with multicomponent diffusion and turbulent combustion module. The OpenFOAM CFD package has been coupled with CHEMKIN II chemistry solving package [20] to accurately calculate the multicomponent thermophysical and transport properties, as well as SUPERTRAPP V3.1 package [21] to determine the nonideal gas properties.

The nonlinear inviscid convective fluxes in AstroFoam are evaluated by the semidiscrete central Kurganov-Tadmor (KT) scheme [22] inherited from rhoCentralFoam [18], which assumes a second-order numerical dissipation in resolving discontinuities (e.g., shock waves and rarefaction tips) yet a high computational efficiency due to its Riemann-free simplicity. The volume integration of the spatial gradient, divergence, and Laplacian terms in the partial differential equations (PDEs) are discretized as the Gauss face integration, where the face values are interpolated by the third-order scale-selective discretization (SSD) scheme [23]. The time integration is advanced by the second-order Crank-Nicolson scheme [24].

The AstroFoam together with the original rhoCentralFoam solver was first validated for various frozen flows, including the canonical shock tube problem, forward step flow, hypersonic flow over a biconic, and supersonic jets $[18,25-31]$. The solver is then applied to various scramjet combustor cases [32-37] to examine its accuracy and robustness in the modeling of complex supersonic combustions.

\section{Governing Equations}

The unsteady and three-dimensional Favre-averaged compressible reactive Navier-Stokes equations (rNSE) are solved for a set of conservative variables $\left(\bar{\rho}, \tilde{u}_{i}, \tilde{H}_{t}, \tilde{Y}_{\alpha}\right)$,

$$
\begin{gathered}
\frac{\partial \bar{\rho}}{\partial t}+\frac{\partial \bar{\rho} \tilde{u}_{j}}{\partial x_{j}}=0 \\
\frac{\partial \bar{\rho} \tilde{u}_{i}}{\partial t}+\frac{\partial \bar{\rho} \tilde{u}_{j} \tilde{u}_{i}}{\partial x_{j}}+\frac{\partial \bar{p}}{\partial x_{i}}-\frac{\partial \tilde{\tau}_{i j}}{\partial x_{j}}=-\frac{\partial \tau_{i j}}{\partial x_{j}} \\
\frac{\partial \bar{\rho} \tilde{H}_{t}}{\partial t}+\frac{\partial \bar{\rho} \tilde{u}_{j} \tilde{H}_{t}}{\partial x_{j}}-\frac{\partial}{\partial x_{j}}\left(\bar{\rho} D_{T} \frac{\partial \tilde{H}_{t}}{\partial x_{j}}+\sum_{\alpha=1}^{L} \bar{\rho} D_{\alpha} \frac{\partial \tilde{Y}_{\alpha}}{\partial x_{j}} \tilde{H}_{\alpha}\right) \\
-\frac{\partial \bar{p}}{\partial t}-\frac{\partial \tilde{u}_{j} \tilde{t}_{i j}}{\partial x_{j}}=-\frac{\partial \Psi_{T, j}}{\partial x_{j}} \\
\frac{\partial \bar{\rho} \tilde{Y}_{\alpha}}{\partial t}+\frac{\partial \bar{\rho} \tilde{u}_{j} \tilde{Y}_{\alpha}}{\partial x_{j}}-\frac{\partial}{\partial x_{j}}\left(\bar{\rho} D_{\alpha} \frac{\partial \tilde{Y}_{\alpha}}{\partial x_{j}}\right)=-\frac{\partial \Psi_{\alpha, j}}{\partial x_{j}}+\bar{\omega}_{\alpha} \\
\bar{p}=\bar{\rho} R \tilde{T}_{t} \\
\tilde{H}_{t}=\tilde{H}+\frac{1}{2} \tilde{u}_{i} \tilde{u}_{i}=\tilde{H}^{0}+\int_{0}^{T} C_{p} \mathrm{~d} T+\frac{1}{2} \tilde{u}_{i} \tilde{u}_{i}
\end{gathered}
$$

Here the bar "-" and the tilde " $\sim$ " represent averaged and Favre-averaged quantities, respectively; $t$ denotes the time; $x_{i}$ is the
Cartesian coordinate in direction $i ; \bar{\rho}$ is the density; $\tilde{u}_{i}$ is the velocity component in $x_{i}$ direction (spatial dimension $i=1,2,3$ ); $\bar{p}$ is the pressure; $\tilde{\tau}_{i j}$ is the viscous stress tensor; $\tilde{H}_{t}=\tilde{H}+0.5 \tilde{u}_{i}^{2}$ is the total absolute enthalpy obtained as the sum of the absolute enthalpy $\tilde{H}$ (sum of the formation enthalpy $\tilde{H}^{0}$ at standard reference state and the sensible enthalpy change going from the reference temperature to $T$ ) and the resolved kinetic energy; $\tilde{Y}_{\alpha}$ is the mass fraction of species $\alpha(\alpha=1, \ldots, L$, where $L$ is the total species number $)$; the specific heat $C_{p}$ is a function of species concentrations and temperature; $\bar{\omega}_{\alpha}$ is the averaged mass production rate of chemical species $\alpha$ in the unit of $\mathrm{kg} \cdot \mathrm{m}^{-3} \cdot \mathrm{s}^{-1} ; D_{\alpha}$ is mixture-averaged mass diffusivity of species $\alpha$; $D_{T}$ is the thermal diffusivity; $\tilde{T}$ is the temperature; $R=R_{u} / W$ is the gas constant; $R_{u}=8.314 \mathrm{~J} \cdot \mathrm{mol}^{-1} \cdot \mathrm{K}^{-1}$ is the universal gas constant; and $W=\left(\sum_{\alpha=1}^{L} Y_{\alpha} / W_{\alpha}\right)^{-1}$ is the molar weight of the multicomponent mixture. The computable averaged momentum diffusive flux is given by,

$$
\tilde{\tau}_{i j}=\bar{\rho} \nu(\tilde{T})\left(2 \tilde{S}_{i j}-\frac{2}{3} \delta_{i j} \tilde{S}_{k k}\right)
$$

which depends on the computable strain-rate tensor of the resolved scales

$$
\tilde{S}_{i j}=\frac{1}{2}\left(\frac{\partial \tilde{u}_{i}}{\partial x_{j}}+\frac{\partial \tilde{u}_{j}}{\partial x_{i}}\right)
$$

The thermodiffusion (Soret effect), barodiffusion, and massdriven diffusion of heat (Dufour effect) are ignored in Eqs. (3) and (4).

The Reynolds stresses $\left(\tau_{i j}\right)$ and turbulent fluxes $\left(\Psi_{T, j}\right.$ and $\left.\Psi_{\alpha, j}^{-}\right)$ in Eqs. (1-6) are unclosed and both require specific modeling. The Reynolds stress, defined as $\tau_{i j}=\bar{\rho}\left(\widetilde{u_{i} u_{j}}-\tilde{u}_{i} \tilde{u}_{j}\right)$, is modeled by the Boussinesq eddy viscosity hypothesis, where the Reynolds stresses are taken to be proportional to the local stain-rate of the resolved flow,

$$
\tau_{i j}=-2 \bar{\rho} \nu_{t} \tilde{S}_{i j}+\frac{2}{3} \delta_{i j} \bar{\rho} k_{t}
$$

Here $\nu_{t}$ is the eddy viscosity given by an approximate turbulence model, and $k_{t}$ is the unresolved turbulent kinetic energy. The turbulent enthalpy flux term $\Psi_{T, j}=\bar{\rho}\left({\widetilde{u_{j} H_{t}}}_{t}-\tilde{u}_{j} \tilde{H}_{t}\right)$ is modeled by the gradient diffusion assumption:

$$
\Psi_{T, j}=-2 \bar{\rho} \frac{\nu_{t}}{P r_{t}} \frac{\partial \tilde{H}_{t}}{\partial x_{j}}
$$

where $P r_{t}$ is the turbulent Prandtl number. The turbulent species diffusion term $\Psi_{\alpha, j}=\bar{\rho}\left(\widetilde{u_{j} Y_{\alpha}}-\tilde{u}_{j} \tilde{Y}_{\alpha}\right)$ is also modeled using the gradient diffusion assumption:

$$
\Psi_{\alpha, j}=-2 \bar{\rho} \frac{\nu_{t}}{S c_{t}} \frac{\partial \tilde{Y}_{\alpha}}{\partial x_{j}}
$$

where $S c_{t}$ is the turbulent Schmidt number.

The Favre-filtered or Favre-averaged Navier-Stokes equations including transport equations for individual species and absolute enthalpy are solved in a uniform framework by equally treating the turbulent viscosity in the RANS mode and the subgrid-scale (SGS) viscosity in the LES mode. The numerical treatments in resolving 
Eqs. (1-11) are the same for any turbulence models, and the switch between RANS or LES is determined by the turbulence model to calculate the eddy viscosity.

The thermodynamic and transport properties of the gas mixture, such as absolute enthalpy, specific heat, viscosity, and thermal and mass diffusivities are calculated using the chemical kinetics package CHEMKIN-II [20] based on the JANAF thermophysical table and a CHEMKIN-format transport database. The viscosity, specific heat, and conductivity depend only on temperature. The mixture-averaged viscosity and thermal conductivity are calculated by the modified Wilke's law [38] and the combination averaging, respectively. The mixture thermal diffusivity is calculated based on the conductivity and specific heat. Mixture-averaged mass diffusivities are used, and the mass conservation is achieved by setting nitrogen as the inert gas.

\section{Turbulence and Combustion Modeling}

To reduce the computational cost in modeling the turbulent boundary layers, IDDES [17] is employed with the background RANS model of one-equation Spalart-Allmaras (S-A) model [39]. In IDDES, the subgrid length scale depends not only on local cell sizes but also on the wall distance, and a shielding function is used to adjust it to avoid the excessively low subgrid viscosity deteriorating the detection of boundary-layer edge. Such a treatment ensures that the boundary layer is fully covered by the RANS mode.

The turbulence-chemistry interaction is accounted for by the partially stirred reactor (PaSR) model [40]. In PaSR, the final averaged reaction rate $\bar{\omega}_{\alpha}$ is determined by the characteristic time scales of chemistry $\left(\tau_{c}\right)$ and micromixing $\left(\tau_{\text {mix }}\right)$,

$$
\bar{\omega}_{\alpha}=\frac{\tau_{c}}{\tau_{c}+\tau_{\text {mix }}} \omega_{\alpha}
$$

where $\tau_{c}$ is the chemical time scale, and $\omega_{\alpha}$ is the reaction rate over the current integration time step calculated from the Arrhenius law. To speed up the direct integration (DI) of the stiff chemistry, in situ adaptive tabulation (ISAT) method [41] is used to calculate $\omega_{\alpha}$. The micromixing time scale approximately equals to the turbulent mixing time scale $\left(\tau_{\mathrm{mix}, t}\right)$, which is defined to be the geometric mean of Kolmogorov time scale $\tau_{k}$ and the SGS time scale $\tau_{\Delta}$ [42] as $\tau_{\text {mix }, t}=\sqrt{\tau_{k} \tau_{\Delta}}$. Here $\tau_{\Delta}=k_{t} / \epsilon=\left(\nu_{t} / C_{\mu} \epsilon\right)^{1 / 2}$ with the constant $C_{\mu}=0.09$, then

$$
\tau_{\mathrm{mix}, t}=\frac{1}{C_{\mu}^{1 / 2}}\left(\frac{\sqrt{\nu \cdot \nu_{t}}}{\epsilon}\right)^{1 / 2}
$$

The above formulation adapts the generic PaSR model for modelings with high mesh resolution. Under laminar or well-resolved DNS-level resolutions, $\nu_{t}=0$, then $\tau_{\text {mix }, t}=0$, and thus $\bar{\omega}_{\alpha} \rightarrow \omega_{\alpha}$, which turns the model back to be the laminar combustion model. The unresolved turbulent kinetic energy $k_{t}$ and its dissipation rate $\epsilon$ are estimated from their relations with the turbulent viscosity $\nu_{t}$, that is, $k_{t}=\left(\nu_{t} /\left(c_{k} d_{\mathrm{IDDES}}\right)\right)^{2}$ and $\epsilon=2 \nu_{\text {eff }}\left|S_{i j}\right|^{2}$, with $\nu_{\text {eff }}=\nu_{t}+\nu$, and $c_{k}=0.07$. The characteristic chemical time scale is calculated as the ratio of the summation of species concentrations to that of forward production rates, $\tau_{c}=\sum c_{\alpha} / \max \left(\sum\left(\omega_{\alpha}^{+} / W_{\alpha}\right), \varepsilon\right)$, with $\varepsilon$ a small quantity to avoid a zero division. The forward production rate $\omega_{\alpha}^{+}$is calculated by only considering the forward reactions and neglecting all their reverse counterparts.

The kerosene combustion reactions are described by a skeletal mechanism consisting of 19 species and 54 elementary reactions [35], which are reduced from the original detailed kerosene mechanism consisting of 2185 species and 8217 elementary reactions [43] using the DRGEPSA method [44]. In the detailed and skeletal mechanisms, the kerosene is surrogated by three components: $28.8 \%$ iso-octane, $62.4 \% n$-decane, and $8.8 \% n$-propylcyclohexane in mass fraction. The accuracy of the skeletal mechanisms has been validated in zero-dimensional and one-dimensional reactors for basic kinetic properties, for example, adiabatic flame temperature, heat release, and ignition delay and laminar flame speed [35].

\section{E. Computational Domain and Boundary Conditions}

The full domain and a quarterly split domain are modeled to compare the influence of symmetry planes. The computational domain consists of three bi-connected sections: isolator, burner, and expander, as denoted in Fig. 1. The unstructured mesh is generated by the Cartesian CutCell method [45], which produces high-quality uniform hexahedral grid cells for more than $98 \%$ of the domain volume in this study, while tetrahedron, wedge, and pyramid cells are filled only in large-curvature regions, for example, those near the borders or corners. As shown in Fig. 2a, a uniform mesh is applied to the whole domain and then adaptively refined based on local curvatures and size functions. The uniform cell size is $0.65 \mathrm{~mm}$ for the quarter domain. For the full domain, three uniform sizes of 1.2, 1, and $0.8 \mathrm{~mm}$ are used to generate the coarse, medium, and fine meshes for grid sensitivity analysis. The cell size around the fuel injectors is progressively refined to $1 / 16$ of the internal cell size, that is, $41 \mu \mathrm{m}$ for $0.65 \mathrm{~mm}, 50 \mu \mathrm{m}$ for $0.8 \mathrm{~mm}, 62.5 \mu \mathrm{m}$ for $1 \mathrm{~mm}$, and $75 \mu \mathrm{m}$ for $1.2 \mathrm{~mm}$, which are all comparable to the local Kolmogorov scale of $50 \mu \mathrm{m}$. The inflation layer comprising 23 prism layers has an average thickness of $2 \mathrm{~mm}$ and the last prism in the logarithmic layer. The initial prism layer's height is $5 \mu \mathrm{m}$, which corresponds to a nondimensional wall distance $y^{+} \sim O(1)$ on all the wall surfaces for the examined combustor flows, as shown in Fig. 2 b. Because the instantaneous $y^{+}$varies both spatially and temporally, to avoid the potential risk of $y^{+}$extruding into the logarithmic layer, an extension of $y^{+}$-insensitive wall treatment is made to the $\mathrm{S}-\mathrm{A}$ model through

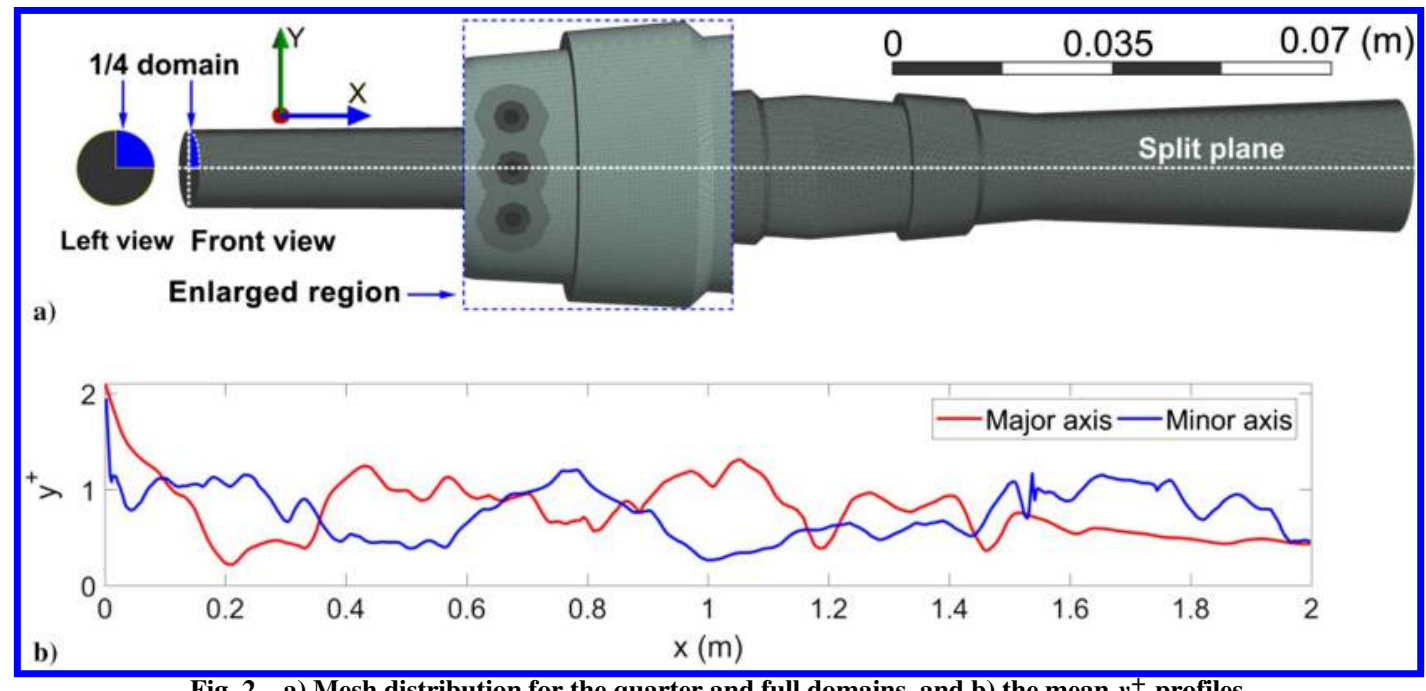

Fig. 2 a) Mesh distribution for the quarter and full domains, and b) the mean $y^{+}$profiles. 
calculating the wall shear stress according to the log-law. The total cell number for the quarter domain is 49.20 million. The coarse, medium, and fine meshes for the full domain, respectively, have 55.35 million, 82.39 million, and 135.60 million cells. The coordinate origin is located at the center of the isolator inlet, and $x$ denotes the streamwise distance from the origin.

Fixed pressure, temperature, velocity, and mixture compositions specified in Table 1 are specified on the inlets of the crossflow and the fuel jet. The principle of extended corresponding states (ECS) for RP-3 kerosene [46] is used to determine the initial fuel jet properties. A RANS-type turbulent inlet boundary condition is specified on the inlets by fixing the turbulence viscosity as $\nu_{t} / \nu=1$. Based on a one-dimensional flow analysis [47], the wall temperature is estimated as a linear variation from $500 \mathrm{~K}$ at the isolator inlet to $1200 \mathrm{~K}$ at the expander outlet to roughly mimic the wall cooling effect. Open boundary condition is applied to the expander outlet, where zero gradient is used for the outflow and the environmental flow conditions are specified when backflow occurs.

It should be noted that updated numerical methods have been implemented in the current modelings compared with the previous one [35]. First, a third-order low-dissipation discretization scheme SSD is applied rather than the traditional second-order total variation diminishing scheme. Second, the heat transfer due to species diffusion is modeled separately, rather than in a whole with the Fourier's law by a constant Prandtl number. Third, the mesh is refined to $1 / 16$ of the internal cell size around the fuel injectors, which is a slight improvement over the previous $1 / 8$.

The parallel computations are performed in TianHe-I cluster with a base CPU frequency of $2.60 \mathrm{GHz}$. For the fine and medium/coarse full-domain modelings, 448 and 280 partitions/cores are used, respectively, whereas 112 partitions/cores are used for the quarterdomain modeling. The time step is limited both by a maximum Courant number of 0.3 and an user-specified maximum time step of $5 \times 10^{-8} \mathrm{~s}$, which is roughly $1 / 10$ to $1 / 20$ of the chemical time scale. The flush through time (FTT) defined based on the length of the combustor flow-path length $(2 \mathrm{~m})$, and the initial flow speed of the vitiated air crossflow $(1501 \mathrm{~m} / \mathrm{s})$ is $1.33 \times 10^{-3} \mathrm{~s}$. Each modeling case costs about $40 \times 24 \mathrm{~h}$ on their assigned core numbers to ensure at least 4 FTTs $(\approx 5 \mathrm{~ms})$ for the data sampling and statistics.

\section{Results and Discussion}

\section{A. Influence on the Mean Flow Fields}

Grid sensitivity analysis has been conducted in the previous study [35] for the quarter domain based on five meshes, and the pressure profiles are re-plotted in Fig. 3a. After applying the updated numerical methods, the current prediction (49.20 million cells) shows a minor difference with the previous ones. For the full-domain modeling, the results predicted by the coarse (55.35 million cells), medium (82.39 million cells), and fine (135.60 million cells) meshes are compared in Fig. 3b. The global error is defined as the normalized absolute deviations from the finest result, $\left|p-p_{135.60}\right| / p_{135.60}$. The maximum global errors are $7.12 \%$ for the coarse mesh and $7.03 \%$ for the medium mesh, whereas the mean global errors are $1.63 \%$ for the coarse mesh and $1.43 \%$ for the medium mesh. The differences between the coarse, medium, and fine predictions are generally small enough to be ignorable. In the following analysis, the results of 49.20 million cells for the quarter-domain modeling and 135.60 million cells for the full-domain modeling are used.

In Fig. 3, two major differences between the quarter-domain and full-domain predictions are that 1) the peak value of 1.85 bar in the full-domain modeling is $11 \%$ higher than that of 1.67 bar in the quarter-domain modeling, and 2) the small initial pressure rise immediately after the flow entry has been completely smeared out in the quarter-domain modeling but is well captured in the full-domain modeling. From $x=0.36$ to $0.66 \mathrm{~m}$, the sharp pressure rise due to the shock-wave/turbulent-boundary-layer interaction (SWTBLI) is not well captured, and all the modelings overpredict the pressure. More dedicated treatments, for example, on the simulation of the boundary layer and the inflow turbulence, may help to improve the prediction of shock impinging behavior and the corresponding pressure rise curve. In comparison with the three repeated measurements, both the quarter-domain and full-domain predictions in the combustor section are satisfactory for the pressure sampling locations from $x=0.66$ to $1.46 \mathrm{~m}$. However, the predictions also suggest that the current distribution interval of pressure transducers is not enough because the peak and some local extremums have not been well captured. Both the quarter-domain and full-domain predictions are slightly higher in the expander section.

Figure 4 shows the three-dimensional distribution of instantaneous and mean Mach number on the symmetry planes and the orthogonal cross sections. From the sonic lines, the instantaneous supersonic core is intermittent although the mean supersonic core is continuous in both the quarter-domain and full-domain predictions. The continuity of mean Mach contours indicates that the two modeled combustors both run in the scramjet mode. The most shrunken supersonic core moves from the downstream cavity in the quarterdomain modeling to the upstream cavity in the full-domain modeling, suggesting that the heat addition occurs more intense when the full domain is applied. The supersonic core in the isolator section has an overall lower mean Mach number for the full-domain modeling. The instantaneous boundary layer in the isolator section is significantly different from the mean one, indicating a considerable unsteadiness for the boundary-layer separation. A much wider subsonic region exists on the major symmetry plane than on the minor symmetry plane, confirming the fact that the convex elliptic section equivalently acts the role of a huge cavity in prolonging the flow residence time. The design concept of "huge cavity" has been proven to be effective in stabilizing the shock train and the flame in a previous analysis [35].

Figure 5a shows the mass-flux-weighted mean temperature on the cross sections perpendicular to the streamwise direction. The medium (82.39 million cells) and fine (135.60 million cells) meshes predict almost identical mean temperature profiles. Before the fuel injection, the temperature is higher in the full-domain modeling; this is because the high-temperature combustion gas propagates more upstream in the full-domain modeling as evidenced by the early initial pressure rise in Fig. 3. After the section with the shortest semi-minor axis at $x=1.02 \overline{5} \mathrm{~m}$, the predicted temperature in the quarter-domain prediction exceeds that in the full-domain modeling.

The cross-section-averaged mean Mach number in Fig. 5b shows that the combustor flow mildly chokes at the fuel injection location in the full-domain modeling while at the downstream cavity in the

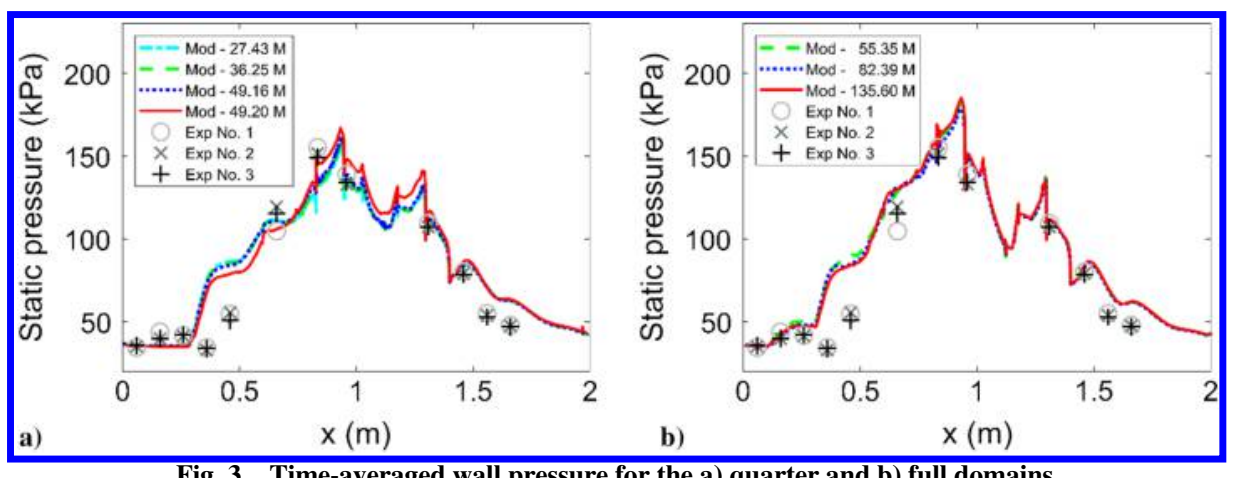

Fig. 3 Time-averaged wall pressure for the a) quarter and b) full domains. 


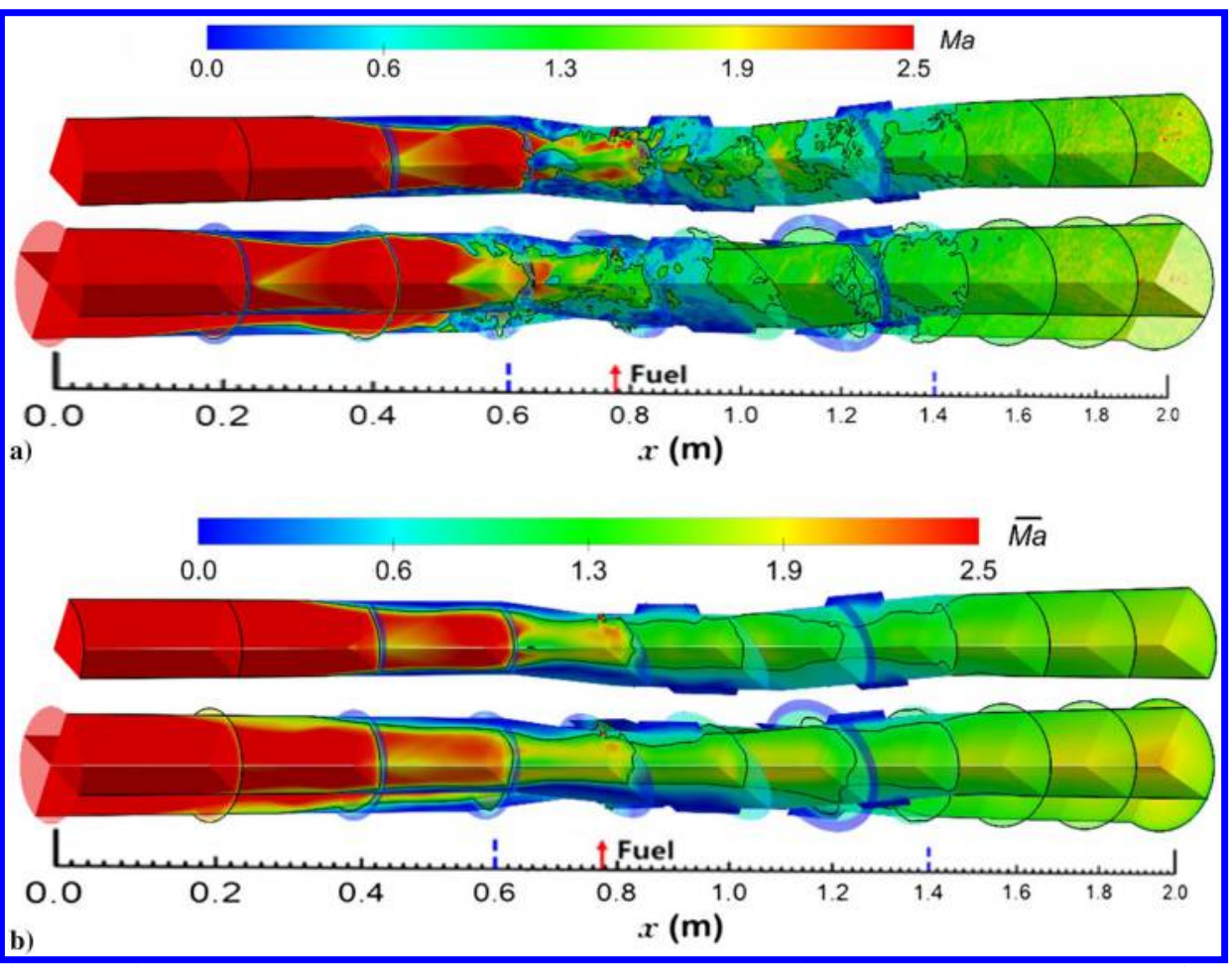

Fig. 4 Contours of a) instantaneous and b) mean Mach number with sonic lines: up, quarter domain; down, full domain.

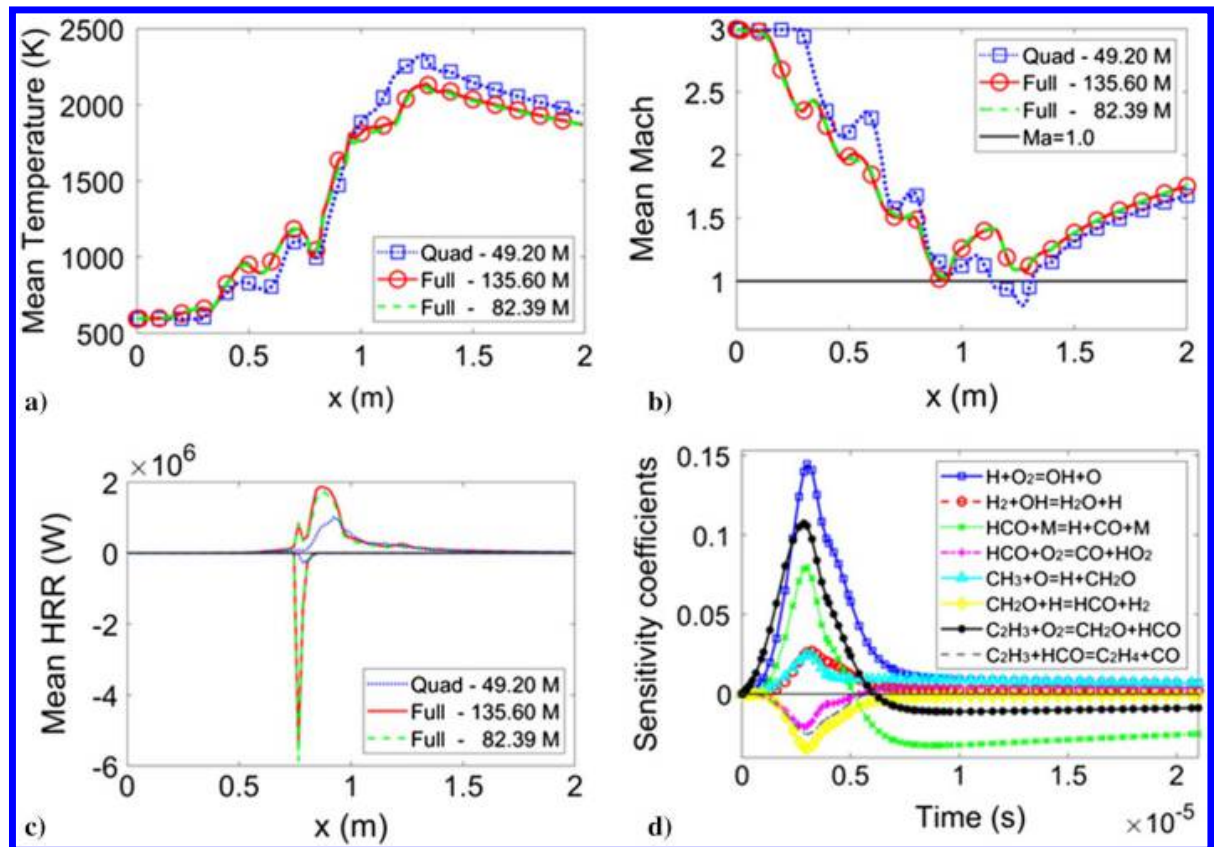

Fig. 5 Streamwise variations of a) temperature, b) Mach number, and c) heat release rate; d) temperature sensitivity coefficients.

quarter-domain modeling. However, the mean Mach number contours shown in Fig. 4 indicate that the modeled combustors both operate in scramjet mode with only slight shrinkage in the supersonic core. The plausible "choking" points in Fig. 5b correspond to the most shrunken supersonic core in Fig. 4, confirming that intense heat addition occurs more upstream in the full-domain modeling. Because of the stronger upstream propagation of the back pressure, the boundary layer is observably thicker in the full-domain modeling, which produces more oblique shock waves and even normal shock waves with bifurcated feet. Consequently, the Mach number in the full-domain modeling varies more smoothly and does not behave the step-like drops as in the quarter-domain modeling.

In Fig. 5c, the positive heat release rate denoting combustion reactions and the negative one denoting pyrolysis reactions are either doubled or of orders of magnitude higher when the full domain is applied. The predicted heat release rate profiles by the medium and fine meshes generally coincide with each other. A close examination shows that the positive peak shifts from the cavity end to the middle of the upstream cavity when using the full domain. The more intense combustion reactions when using a full domain could be related to the bilateral exchanges of high-temperature gas and active radicals across the symmetry planes. The peak pressure in Fig. 3 rises accordingly due to the more intense heat release in the full-domain modelings. The negative peak that is of orders of magnitude higher in the fulldomain modeling can be attributed to the raised temperature around the fuel injectors. Because of the wider existence of recirculation flows near the wall in the full-domain modeling, the fuel is preheated by its downstream burning. For the same reason, the negative peak 
occurs more upstream for the full-domain modeling. From Fig. $\underline{\text { c }}$, there is a secondary positive peak corresponding precisely to the negative peak and is only observed in the full-domain modeling.

Figure $5 \mathrm{~d}$ shows the normalized sensitivity coefficients as a function of time for the eight reactions that have the most significant effect on the gas temperature. The coefficients are calculated during the autoignition of a homogeneous stoichiometric mixture of kerosene and air under constant pressure of 1 bar and an initial temperature of $2400 \mathrm{~K}$. The positive sensitivity coefficient indicates that increasing the rate of this reaction will lead to a higher temperature (more heat production), and vice versa. The sensitivity coefficients of the oxidation reactions near the ignition time are much larger than those of the pyrolysis reactions in the initial reaction stage, but this does not mean that the pyrolysis reactions are not important, but just because the temperature change during the initial pyrolysis stage is not as rapid as that near the ignition. As seen, in the ignition stage, there are both endothermic and exothermic reactions. The dominant reaction that increases the mixture temperature is a chain-branching step, $\mathrm{H}+\mathrm{O}_{2}=\mathrm{OH}+\mathrm{O}$, and the dominant contributor to the negative heat release rate is the $\mathrm{H}$-abstraction reaction of formaldehyde, $\mathrm{CH}_{2} \mathrm{O}+\mathrm{H}=\mathrm{HCO}+\mathrm{H}_{2}$. There are some reactions, for example, $\mathrm{HCO}+\mathrm{M}=\mathrm{H}+\mathrm{CO}+\mathrm{M}$ and $\mathrm{C}_{2} \mathrm{H}_{3}+\mathrm{O}_{2}=\mathrm{CH}_{2} \mathrm{O}+\mathrm{HCO}$, whose reverse reaction may become important as the concentrations of the product species increase in the postignition stage. It is suspected that those reactions with positive coefficients in the ignition stage and negative values afterward may be the cause for the secondary peak in Fig. 5c. The fact that no apparent secondary peak is observed for the quarter-domain modeling could be attributed to the weak ignition reactions with the lack of preheated crossflow and exchanged radicals.

As expected, the instantaneous flow is asymmetric as indicated by the sonic lines depicted on the instantaneous velocity field in Fig. 6a. In addition to the supersonic core, the boundary-layer separation on the major plane is apparently asymmetric. The streamlines drawn based on the mean velocity in Fig. $6 \mathrm{~b}$ show that the flow symmetry is better on the minor symmetry plane but has been obviously broken on the major symmetry plane. The most asymmetric flow occurs in the combustor section before the end of the upstream cavity, where most of the heat release takes place. In addition to the instability arisen by the volume expansion, the upstream propagation of back pressure causes severe asymmetric boundary-layer separation in the isolator section before the shape inflection point at $x=0.6 \mathrm{~m}$. As evidenced by the mean streamlines, a small flow recirculation has occurred inside the upper boundary layer on the major symmetry plane. In most of the flow path, the boundary layer may remain attached and merely be thickened by the adverse pressure rise. A thickened boundary layer would always facilitate its further thickening because the viscous force has been strengthened through the broadened undulating contact interface between the boundary layer and the supersonic core. For supersonic boundary layer, the interface can be identified as the sonic isosurface. The consequence of such feedback is that the thickening of a boundary layer may accelerate, to the extent that an asymmetric boundary-layer separation can be visibly observed, as evidenced in this study. An over-thickened boundary layer usually drills more upstream adhering to the wall. When the boundary layer on one side is over-thickened, its counterpart on the opposite side will become even thinner than the case in a symmetry domain modeling, being referred to as an under-thickened boundary layer. It is interesting to observe that the over-thickening and underthickening boundary layers may appear alternately on the same side, with the underlying mechanism is unknown. The high-temperature gas convected upstream by the flow circulations in the thickened boundary layer may cause asymmetric ablation on the combustor walls as observed during the experiments.

Previous studies $[\underline{8}, \underline{48}]$ indicate that when the area-expansion ratio $(A R)$ exceeds 1.5 , significant asymmetry with two unequal recirculation zones will emerge for flows experiencing a sudden expansion. The area-expansion ratio, defined as $A R=1+h / W_{0}$, is calculated as $A R=1.57$ for this case using the diameter of the burner inlet as $h$ and the longest major axis in the burner section as $W_{0}$. Such a high AR suggests that the flow symmetry should have been broken both instantaneously and in the mean. The underlying reason for the asymmetry under steep backward facing step is attributed to the Coanda effect, that is, the tendency of a jet to attach itself to an adjacent wall surface due to the lower pressure developed by entraining fluid from the surroundings. Thus it is suggested that a reduction in the major axis may help to increase the flow symmetry.

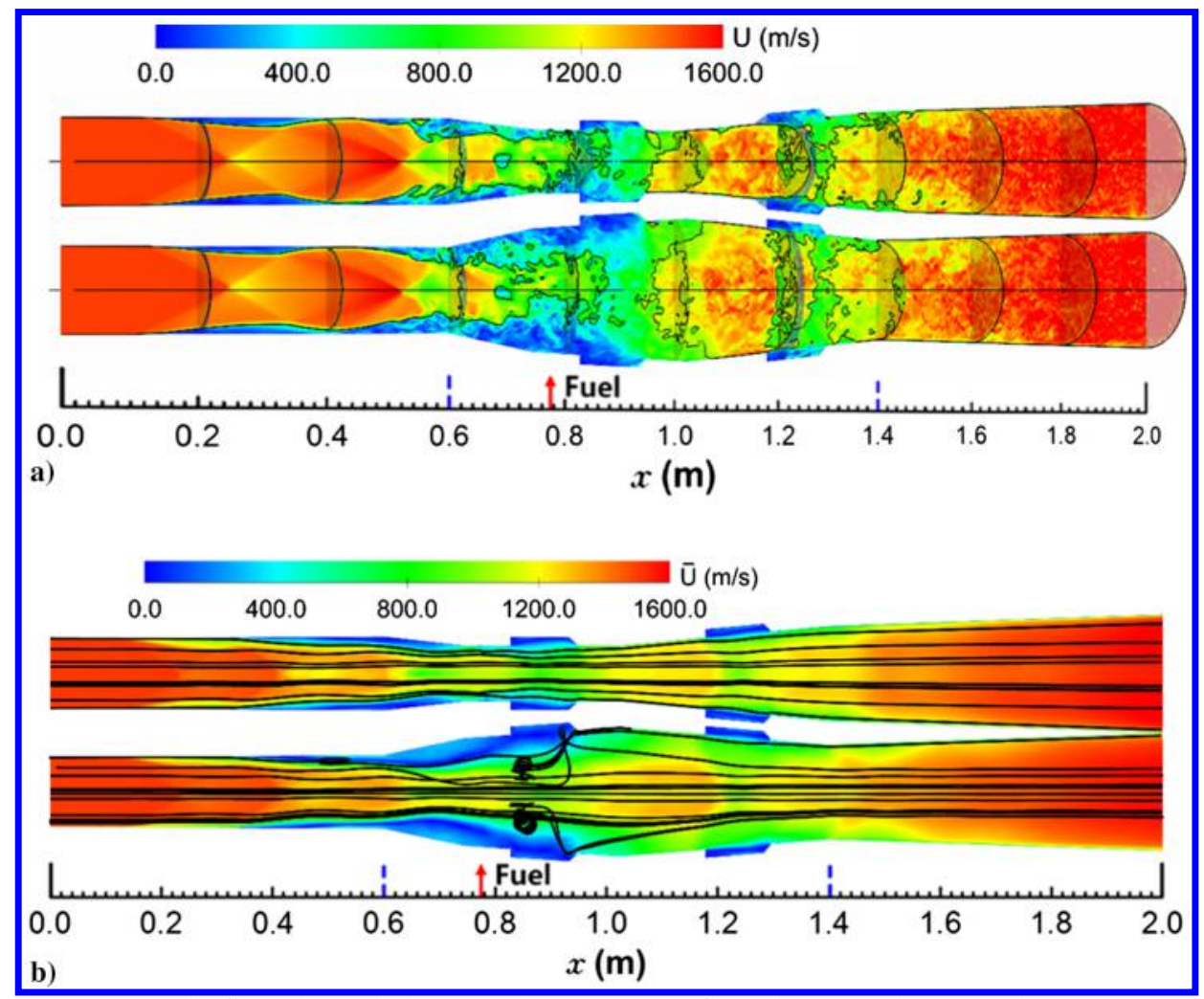

Fig. 6 a) Instantaneous velocity field with sonic lines, and b) mean velocity field with streamlines; up, minor plane; down, major plane. 


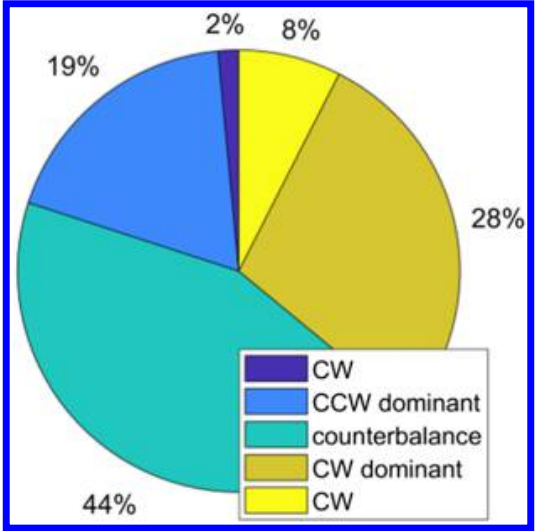

Fig. 7 Streamwise statistics of the flow swirling directions.

Further splitting the major and minor symmetry planes by the central axis, the time-averaged mass flow rates through the four half-planes over the 4 FTTs are 1) 0.0044 and $-0.0107 \mathrm{~kg} / \mathrm{s}$, respectively, through the upper and lower halves of the minor symmetry plane, and 2) -0.0028 and $0.0082 \mathrm{~kg} / \mathrm{s}$, respectively, through the upper and lower halves of the major symmetry plane. Here the upper half-planes are on the positive half-axes, and a positive flow rate is defined to head the positive orientation. Thus the sign order of $[+,-,-,+]$ for the integral mass flow rates indicates a clockwise swirling flow if viewing in the $+x$ direction. Statistics on the flow rotating direction at different streamwise locations are summarized in Fig. 7, which shows that the clockwise (CW) swirling is dominant over the counterclockwise (CCW) swirling and an observable symmetry breaking has occurred.

\section{B. Flow Exchanges Across the Symmetry Planes}

Figure 8 shows the instantaneous temperature field on the minor and major symmetry planes in the full-domain modeling. The instantaneous distributions temperature and the mixture concentration denoted by the stoichiometric line are far from symmetry. The mixing between the fuel jet and the vitiated air crossflow seems to be reasonably good behind the upstream cavity, where the temperature is also favorable for the combustion reactions. There are evident exchanges of hot combustion gas and highly reactive mixture parcels across the symmetry planes.

Figure 9 shows the relative probability of pressure and temperature statuses based on a historical statistic of the instantaneous fields over the 4 FTTs. The relative probability counts the probability of data points falling into a subspace of $3 \mathrm{kPa} \times 30 \mathrm{~K}$, and the sum of all the relative probabilities in the examined pressure-temperature space of 3-1020 kPa $\times 300-2790 \mathrm{~K}$ is unity. The data are resampled using a uniform spatial grid of $1 \mathrm{~mm} \times 1 \mathrm{~mm}$ to avoid the aliasing error caused by nonuniform cell sizes. As seen, there are two main streak-like clustering regions. The lower one corresponds to the fuel-depletion crossflow, where the pressure rise is mainly due to compression, and the temperature rise is due to aerodynamic heating and viscous heating. The upper one corresponds to the burning fuel mixture, which has a mixture fraction higher than the stoichiometric

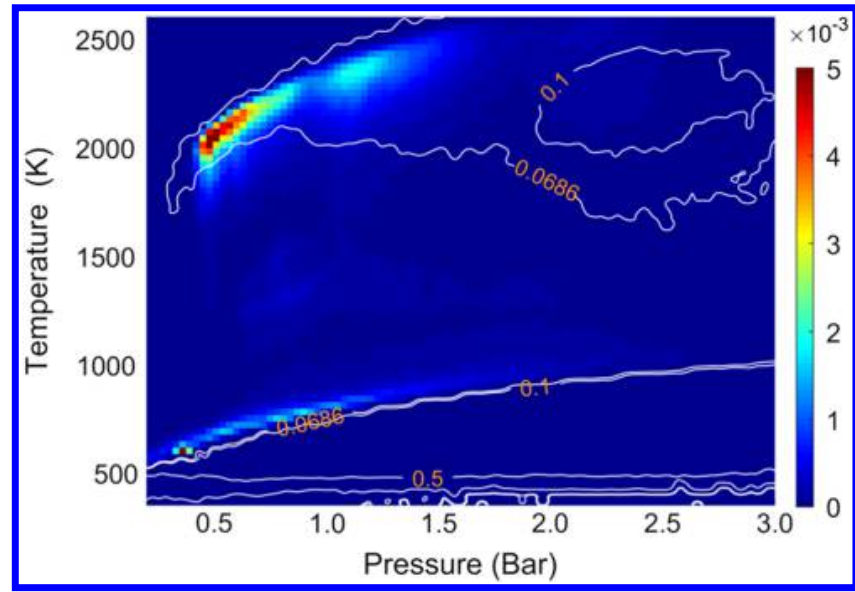

Fig. 9 Relative probability of pressure-temperature statuses contoured by mixture fraction.

value of $\xi_{\mathrm{st}}=0.0686$. One noteworthy observation from the mixture fraction contours is that the peak temperature occurs always on the fuel-rich side, which is the well-known rich-shifting phenomenon [49]. Considering the rich flammability limit RFL $=2 \xi_{\mathrm{st}}$, most of the reaction zones are flammable. The typical temperature and pressure ranges in the reaction zones of the current scramjet combustor are $2000-2500 \mathrm{~K}$ and $0.5-1.8$ bar.

Three typical chemical statuses sampled on the upper ridge (corresponding to the most active reaction zone) of the probability distribution are extracted to draw the diagrams of reaction path in Fig. 10. The pressure, temperature, and mixture fraction for the three statuses are, respectively, $0.5 \mathrm{bar} / 2056 \mathrm{~K} / 0.0786$ (denoted by status 1), $1 \mathrm{bar} / 2297 \mathrm{~K} / 0.0823$ (status 2), and $1.5 \mathrm{bar} / 2511 \mathrm{~K} / 0.0810$ (status 3 ). The species composition is sampled at the matched cell that has the most closest pressure, temperature, and mixture fraction with the selected status. The path diagrams of statuses 2 and 3 are the same except with different path fluxes, whereas the path diagram of status 1 is markedly different from the other two. The path flux is calculated as the summation of production and consumption fluxes [50]. The line thickness is proportional to the path flux normalized by the maximum path flux in the current diagram. The reaction paths with normalized fluxes smaller than $1 \times 10^{-10}$ are ignored for clarity. The reaction path from $\mathrm{CO}$ to $\mathrm{CO}_{2}$ has an overwhelmingly higher reaction rate over the others for all the three statuses. All the three reaction path networks start from $\mathrm{C}_{3} \mathrm{H}_{6}$ and end at $\mathrm{CO}_{2}$. The key species are those that are responsible for the main consumption of $\mathrm{C}_{3} \mathrm{H}_{6}$ or the main production of $\mathrm{CO}_{2}$. The key species are identified as $\mathrm{C}_{2} \mathrm{H}_{3}, \mathrm{CH}_{3}, \mathrm{HCO}$, and $\mathrm{CO}$ for status 1 , and as $\mathrm{C}_{2} \mathrm{H}_{4}, \mathrm{CH}_{2} \mathrm{O}, \mathrm{HCO}$, and $\mathrm{CO}$ for statuses 2 and 3 . Some species act as key junctions in the whole path networks, that is, $\mathrm{CH}_{2} \mathrm{O}$ and $\mathrm{C}_{2} \mathrm{H}_{4}$ for status 1 , while $\mathrm{C}_{2} \mathrm{H}_{3}$ and $\mathrm{CH}_{3}$ for statuses 2 and 3. The large deficits in the production and consumption fluxes of those junctional species imply that they are short-lived. The temperature sensitivity analysis in Fig. 5d has emphasized the importance of $\mathrm{C}_{2} \mathrm{H}_{3}, \mathrm{CH}_{3}, \mathrm{CH}_{2} \mathrm{O}$, and $\mathrm{HCO}$ in determining the heat release rate. With the pressure rises by $50 \%$ from statuses 2 to 3 , most of the path fluxes increase

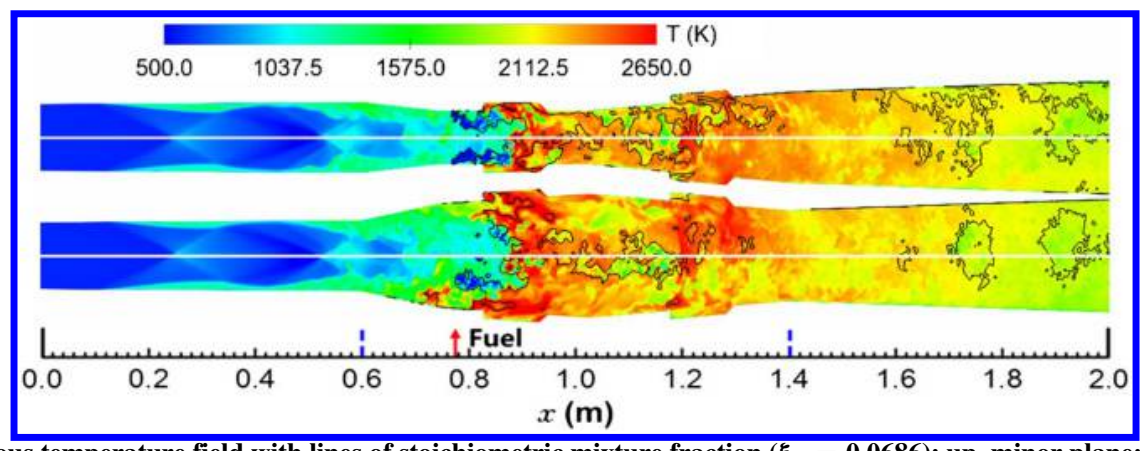

Fig. 8 Instantaneous temperature field with lines of stoichiometric mixture fraction $\left(\xi_{s t}=0.0686\right)$ : up, minor plane; down, major plane. 


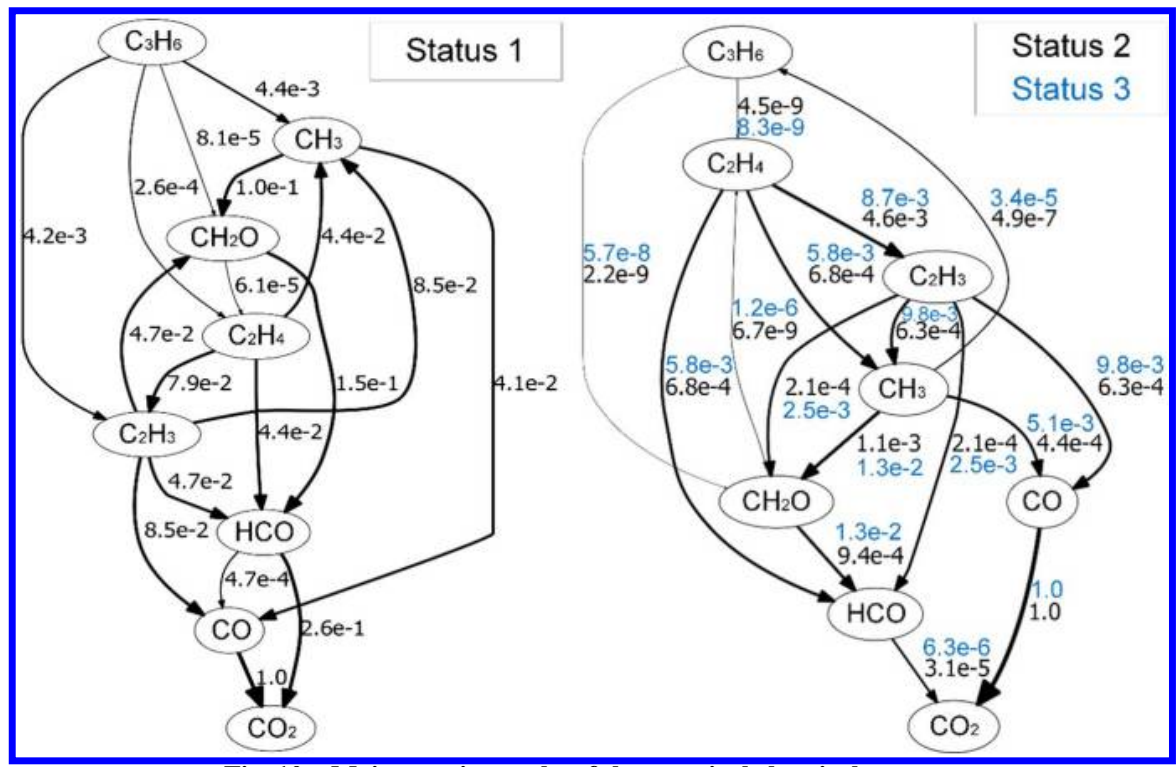

Fig. 10 Main reaction paths of three typical chemical statuses.

by at least one order of magnitude. From status 1 to statuses 2 and 3, the path from $\mathrm{C}_{3} \mathrm{H}_{6}$ to $\mathrm{C}_{2} \mathrm{H}_{4}$ becomes more important. As seen, the path diagram from the fuel molecules to the final products $\left(\mathrm{CO}_{2}\right.$ and $\left.\mathrm{H}_{2} \mathrm{O}\right)$ varies both spatially and temporally, suggesting that the skeletal mechanism should also be adaptively changed to best describe the local chemistry as well as to minimize the mechanism size.

The exchanges of mass, high-temperature gas, force, and oxygen across the symmetry planes along the streamwise direction are presented in Fig. 11. The force is calculated as the surface integration of momentum flux and then scaled by the value on the isolator inlet. The exchange of high-temperature gas is estimated by the net mass flow rate (in $\mathrm{kg} / \mathrm{s}$ ) of mixture parcels with a temperature higher than $1650 \mathrm{~K}$, which corresponds to an ignition delay short enough to allow the mixture to be ignited within the maximum transverse distance (the domain width). The mass exchanges of the flow and high-temperature gas are scaled by the total mass flow rate from the inlets of the isolator and the fuel injectors, whereas the mass exchange of each species is scaled by its mass existence in the whole domain divided by 1 FTT. The exchanges of mass flow rate shown in Figs. $11 \mathrm{a}$ and $11 \mathrm{~b}$ are small quantities compared with the incoming mass flow rate. Thus from the perspective of mass conservation, the flow symmetry can be considered to be reasonably good over the 4-FTT integration. The mass inflow (flow in the positive orientation) and outflow exhibit a spatial quasi-periodicity; that is, the dominance of inflow and outflow changes approximately every $10-15 \mathrm{~cm}$. The flow first becomes sinuous in the isolator, as evidenced by the absolute dominance of inflow or outflow before $x=0.6 \mathrm{~m}$, and then the flow direction becomes stochastic as neither inflow nor outflow diminishes to a very low level. On the major symmetry plane, the outflow rate rises remarkably at the trailing edge of the upstream cavity, which indicates that the core flow has been slightly deflected by the volume expansion force arisen from the intense heat release. The distributions of scaled force in Figs. 11c and 11d exhibit high proportionality with the scaled mass flow rate, which suggests that the imbalance of momentum exchange drives the mass flow. The underlying difference between a quarter-domain and the full-domain internal combustion is that the high-temperature gas and active radicals entrained by the cross-border mass flow, even in a very small quantity, can help to ignite and stabilize the flame on the opposite side. That is the reason for a more strengthened heat release rate as in Fig. 5c. As in Figs. 11e and 11f, the high-temperature gas crossing the minor symmetry plane is roughly balanced, indicating good energy conservation. Across the major symmetry plane, the interaction between the two rows of jet flames in a "counterdiffusion" layout is more intense, and the transport of high-temperature gas is biased because of the deflected core flow. Similarly, the oxygen exchange across the minor symmetry plane in Figs. $11 \mathrm{~g}$ and $11 \mathrm{~h}$ is overall balanced in the burner section, regardless of the drastic undulations caused by the sinuous core flow in the isolator section. The overwhelming influx of oxygen into one single side implies that the reactions there are more violent and accordingly the expansion force points to the opposite side.

Figure 12 compares the scaled imbalances of mass, force, hightemperature gas, and species across the two symmetry planes. Across the minor symmetry planes, the flow direction of fuel species $\left(\mathrm{NC}_{10} \mathrm{H}_{22}, \mathrm{IC}_{8} \mathrm{H}_{18}\right.$, and $\left.\mathrm{PCH}\right)$ is in reverse with almost all the other species, which indicates that the fuel jets tilt slightly to one side and the burnt mixture is then delivered back again. Across the major symmetry plane, the exchanges of unburnt fuel species are small while all the other species warped up in the burnt mixture are driven, possibly by the volume expansion force, along the reverse direction of the oxygen replenish flux. Those species among the highest rank of imbalance are $\mathrm{C}_{2} \mathrm{H}_{3}, \mathrm{C}_{2} \mathrm{H}_{4}, \mathrm{CH}_{2} \mathrm{O}, \mathrm{HCO}$, and $\mathrm{CO}$, which are all key species from the previous reaction path analysis in Fig. 10. In the quarter-domain modeling, the autoignition occurs far downstream of the fuel injection because it takes time for the radical farming [51]. However, in the full-domain modeling, the bilateral exchanges of key species increase the path fluxes by orders of magnitude, and a mutually aided ignition mechanism is hence established. The strengthened downstream combustion reactions can be attributed to both the early ignition (registered by the secondary peak in Fig. 5c) and the ongoing species exchanges. $\mathrm{H}_{2}, \mathrm{H}$, and $\mathrm{OH}$, which have high temperature sensitivity coefficients in Fig. 5d, also have considerable exchange imbalances. Because of their high activity, $\mathrm{H}_{2}$ and $\mathrm{H}$ are short-lived and thus are absent in most of the in situ reaction path diagrams. The nitrogen, total mass, high-temperature gas, and force all have relatively small exchange imbalances, which demonstrates that the ensemble flow has statistically symmetric distribution in mass, momentum, and temperature, although the exchanges of individual species may be heavily unbalanced.

Figure 13 compares the mixing and combustion efficiencies as well as the total pressure loss for the quarter-domain and full-domain modelings following the definitions in [52]. The time-averaged mixing efficiency is calculated as,

$$
\eta_{\text {mix }}=\frac{1}{n} \sum_{n} \frac{\dot{m}_{\text {fuel,mixed }}}{\dot{m}_{\text {fuel,total }}}=\frac{1}{n} \sum_{n} \frac{\int\left(\rho \boldsymbol{u} Y_{f, \text { react }}\right) \cdot \mathrm{d} \boldsymbol{A}}{\int\left(\rho \boldsymbol{u} Y_{f}\right) \cdot \mathrm{d} \boldsymbol{A}}
$$

with

$$
Y_{f, \text { react }}= \begin{cases}Y_{f} & Y \leq Y_{f, \mathrm{st}} \\ Y_{f, \mathrm{st}} \frac{1-Y_{f}}{1-Y_{f, \mathrm{st}}} & Y>Y_{f, \mathrm{st}}\end{cases}
$$



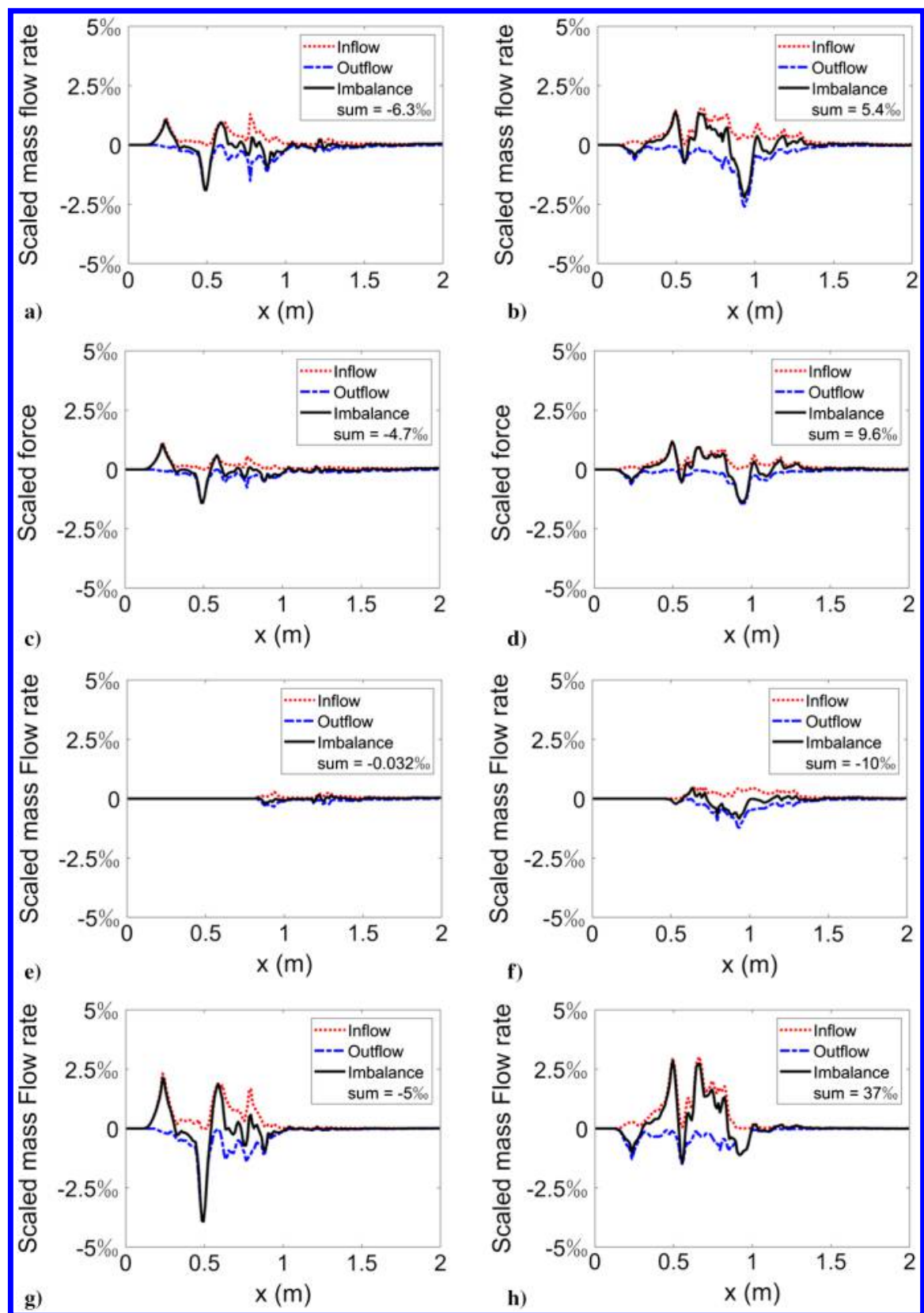

Fig. 11 Bilateral exchanges of a, b) mass; c, d) force; e, f) high-temperature gas; and g, h) oxygen; a, c, e, and g, minor plane; b, d, f, and h, major plane.

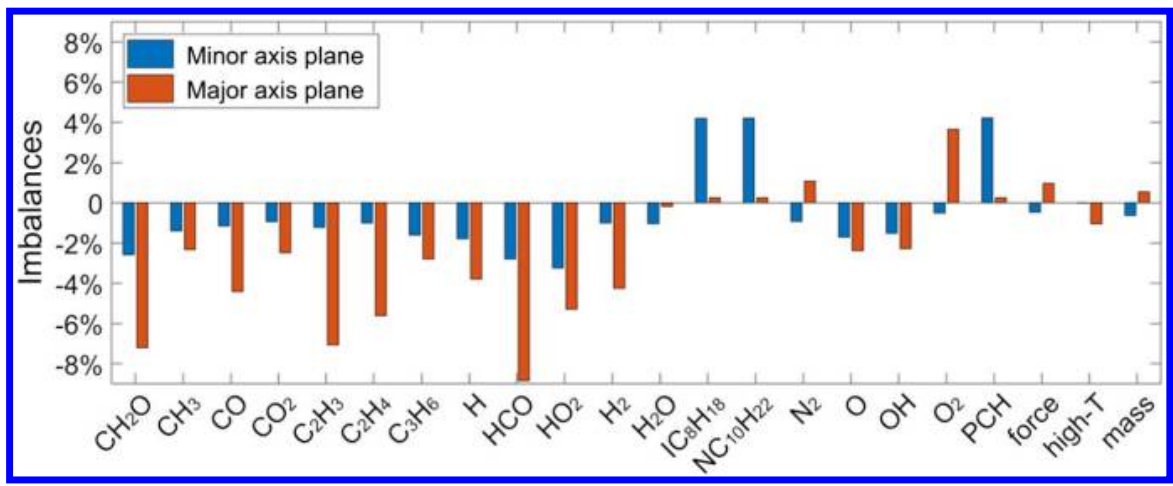

Fig. 12 Ensemble exchanges of mass, force, high-temperature gas, and species.

where $\dot{m}_{\text {fuel,mixed }}$ and $\dot{m}_{\text {fuel,total }}$ are the mass flow rates of mixed and total fuel, $Y_{f}$ is the fuel mass fraction, $Y_{f \text {,react }}$ is the mass fraction of the fuel that can be reacted, and $Y_{f, s t}$ is the fuel mass fraction at the stoichiometric condition. Note that $Y_{f}$ is derived from the mixture fraction based on the element fractions following the Bilger's definition [53] to adapt Eq. (14) for both nonreacting and reacting 


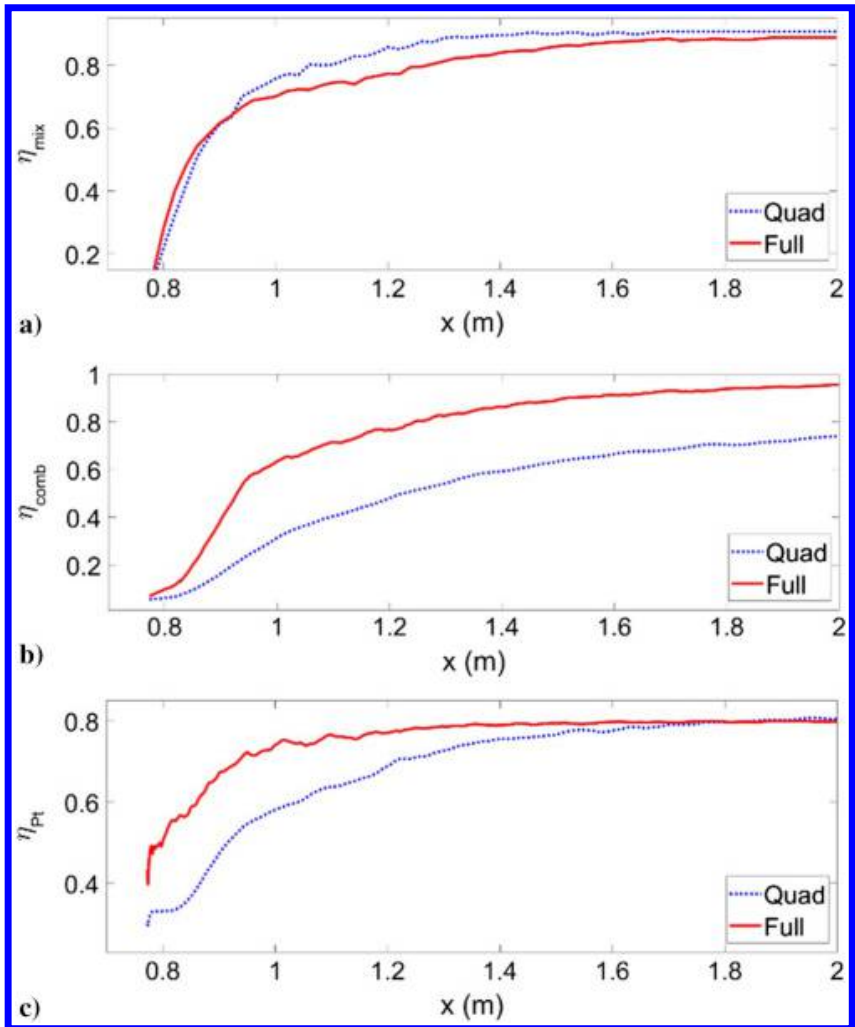

Fig. 13 a) Mixing efficiency, b) combustion efficiency, and c) total pressure loss $C$. Influence on turbulence and turbulence-chemistry interaction.

cases. Combustion efficiency is calculated as the ratio of the fuel that has been completely converted to the final stable products, for example, $\mathrm{CO}_{2}$ :

$$
\eta_{\text {comb }}=\frac{1}{n} \sum_{n} \frac{\left[\left(1 / \nu_{\mathrm{CO}_{2}}\right) \int\left(\rho \boldsymbol{u} Y_{\mathrm{CO}_{2}}\right) \cdot \mathrm{d} \boldsymbol{A} / W_{\mathrm{CO}_{2}}\right]_{\text {inlet }}^{x}}{\dot{m}_{\text {fuel,total }} / W_{\text {fuel }}}
$$

where the subscript inlet denotes variables on the inlet plane, the surface integration is performed on each cross section at different streamwise locations, $Y_{\mathrm{CO} 2}$ is the mass fraction of $\mathrm{CO}_{2}, W$ is the molecular weight of the fuel or $\mathrm{CO}_{2}$, and $\nu_{\mathrm{CO}_{2}}$ is the stoichiometric coefficient of $\mathrm{CO}_{2}$. The total pressure loss is defined as,

$$
\eta_{P_{t}}=1-\frac{1}{n} \sum_{n} \frac{\int\left(\rho \boldsymbol{u} P_{t, x}\right) \cdot \mathrm{d} \boldsymbol{A}}{\int\left(\rho \boldsymbol{u} P_{t, \text { inlet }}\right) \cdot \mathrm{d} \boldsymbol{A}}
$$

where $P_{t, x}$ and $P_{t, \text { inlet }}$ are the total pressure at the streamwise location $x$ and the inlet plane, respectively.

The profiles of mixing efficiency in Fig. 13a for the two domains are similar. The full-domain modeling predicts a slightly lower final efficiency of 0.89 compared with the value of 0.91 for the quarter-domain modeling. The mixing efficiency in the full-domain modeling initially rises quickly, which can be due to the enhanced macrostirring arisen by the more intense yet more concentrated heat release. A noticeable difference is for the combustion efficiency in Fig. 13b, where the full-domain modeling predicts an increment of $0 . \overline{25}$ over the quarter-domain prediction. The final combustion efficiency of 0.95 implies an almost complete fuel consumption when no symmetry assumption has been applied, in contrast to the relatively low value of 0.74 in the quarter-domain modeling. The exchanges of high-temperature gas and active radicals do strengthen the autoignition and combustion reactions on the two sides. Another explanation for the increased combustion efficiency in the full-domain modeling is the species defect effect. The multicomponent diffusion and flux discretization may cause the defect in the intermediate species, leading to incomplete chain reactions. The case is especially worse for skeletal mechanisms because the role of certain intermediate species may become prominent with the simplification of the reaction paths. However, the phenomenon of species defect can be significantly alleviated for the lumped reaction zone in the full-domain modeling, as most of the reaction process can be completed locally. The total pressure loss in Fig. $13 \mathrm{c}$ has a drastic increment immediately after the fuel injection once the full domain has been applied, but the final losses for the two domains are almost identical with all approaching 0.8 . The increased total pressure loss in the burner section for the full-domain modeling can be partially attributed to the Rayleigh heating effect because the combustion efficiency and the heat release rate increase considerably. On the other hand, the size of the largest eddies in the full domain is larger than that in a split domain. Therefore the strengthened turbulence in the full domain with a doubled Reynolds number $(R e)$ will further increase the entropy and reduce the total pressure loss.

Figures 14 shows the vortex structures around the fuel injectors in the quarter-domain and full-domain modelings. Unlike the typical vortexes in nonreacting jet in supersonic crossflow (JISC) [54], the counterrotating vortexes (CRV) are much short with the $\Omega$-shape vortexes obscured by the thermal expansion in the current reacting JISC cases. However, clear horseshoe vortexes on the upwind of the fuel jets can still be observed. Not only the size of the largest vortexes is confined by the width of the quarter domain, but also the vortexes close to the symmetry planes are obviously influenced. The vortex structures around the split injector in Fig. 14a are less richer than its complete counterpart in Fig. 14b, and also less than its neighbor injector in Fig. 14a. An effective CRV cannot be formed due to the lack of pairing, and circumferential rollers cannot be fully developed into the $\Omega$-shape vortexes. Large-scale transverse vortexes are truncated by the symmetry planes, and an effective vortex stretching cannot take place. Consequently, the vortexes around the split
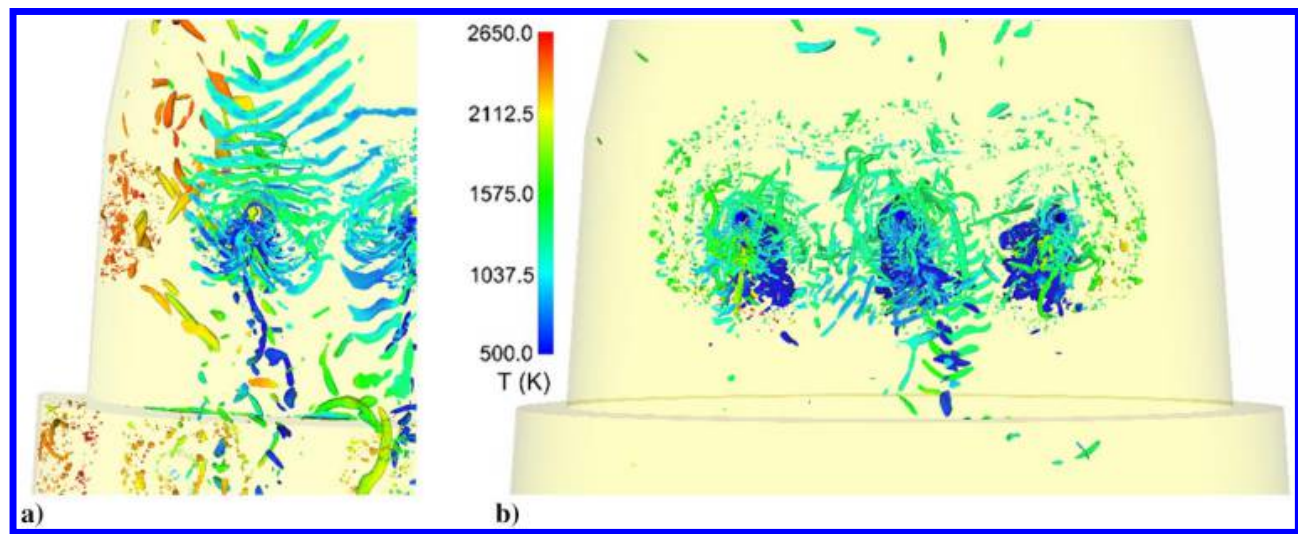

Fig. 14 Vortex structures represented by the iso-surfaces of Q-criterion (the second invariant of the velocity gradient tensor) at $2 \times 10^{10} \mathrm{~s}^{-2}$ and colored by temperature: a) quarter domain; b) full domain. 


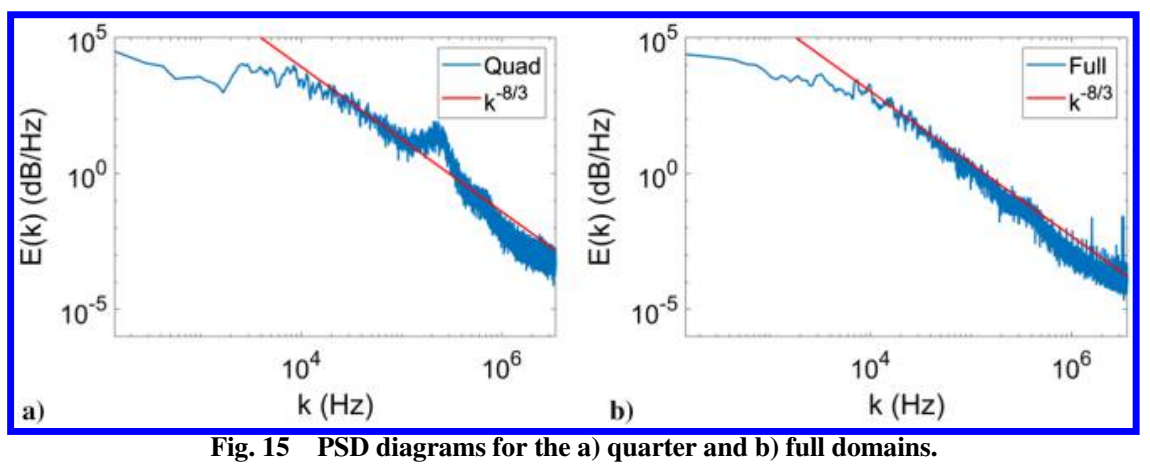

injector are in smaller sizes and mostly align with the streamwise direction.

Figure 15 shows the power spectral density (PSD) diagrams extracted from the temporal sampling of static pressure at the center of the exit plane of the burner section $(x=1.4 \mathrm{~m})$, which is in the LES-resolved flow portion. For the quarter domain, the symmetry equivalently acts as a slip wall, where the lateral motions normal to the symmetry planes are restricted. Turbulence confined by walls is no longer well approximated as isotropic and homogeneous, and so the dimensional argument leading to the Kolmogorov $k^{-5 / 3}$ slope ( $k$-frequency or wave number) lose their validity and the associated inertial subrange spectra cannot be expected a priori [55]. A shorter inertial subrange is observed for the quarter-domain modeling compared with the full-domain modeling, which is partially due to the influence of the symmetry boundaries. Another explanation for the shorter inertial subrange can be due to the decreasing of integral-scale $R e$ in the quarter-domain modeling. For both the quarter-domain and full-domain results, the dependence of energy density $E(k)$ at each frequency $k$ is fit to be $E(k) \sim k^{-8 / 3}$, rather than the exponent of $-5 / 3$ in subsonic flows. The exponent of $-8 / 3$ has been previously obtained in [56] for supersonic flows, where the compressibility significantly affects the transfer of kinetic energy from larger to smaller scales. Other slopes steeper than the Kolmogorov's $-5 / 3$ have also been reported [57-60] and the underlying reason is attributed to the weakened role of the convective transport and vortex stretching terms due to the nearly alignment of the vorticity and velocity vectors in the supersonic regions far from shocks and walls. The steeper slope means that energy starts to be dissipated at lower wavenumbers as the dissipative eddies are larger in supersonic flows [56], which is also justified by the fact that the viscous dissipation scales as $M a^{2} / \operatorname{Re}$ [57]. This is a good news because it means that the smallest eddies may actually be larger than the flame front thickness and therefore they cannot penetrate into the flame front to disturb the local reactions; consequently, a decoupling treatment of chemistry modeling and flow modeling can greatly accelerate the turbulent combustion modeling.

In the quarter-domain modeling, a unique spike structure exists to bridge the inertial subrange and the viscous dissipation subrange, which indicates that a direct interaction across the scales near the spike exists with significant energy transfer to the small as well as the large scales [61]. Similar spikes in the kinetic energy spectra were observed in [6] $]$, which pointed out that energy is not only cascaded in the inertial subrange but can also be directly extracted by imposed forces and then transferred into larger scales. In supersonic combustion, the imposed forces can be from the discontinuities like shock waves and eddy shocklets, as well as the thermal expansion produced by the chemical heat deployed in the flame. Possibly the dominant imposed force in the current case originates from the symmetry boundary conditions implemented in the quarter-domain modeling. The imposed force causes a strong anisotropic effect that enables one component of the SGS stress to supersede the isotropizing restoration [12] and consequently renders backscatter of kinetic energy into larger scales. Note that combustion can also deploy extra kinetic energy into larger scales through pressure-dilatation work and flows driven by flame-front instabilities $[11,63]$. The spectrum spike is indeed an energy accumulation phenomenon formed by the backscatter energy from the smaller scales and the forward scatter energy from the inertial subrange. A less obvious cusp can also be observed at the end of the inertial subrange for the full-domain modeling, which highlights the importance of the external force imposed by the symmetry planes, and also suggesting that the SGS turbulence is still strongly anisotropic due to some other forces imposed by compressibility and combustion at the sampling point. The spectrum is then truncated at a high frequency in the dissipation range by the sampling rate of around $2 \times 10^{7} \mathrm{~Hz}$.

As evidenced by the resolved flow results, the backscatter of kinetic energy can become prominent in high-Ma turbulent reacting flows $[12,63]$. Usually, the backscatter and the countergradient diffusion of scalars occur simultaneously, which implies that non-Boussinesq transport typically characterized by a negative eddy viscosity should be reflected in the turbulence model. However, due to the inherent complexity of the SGS closure problem, no sophisticated turbulence models have been developed to well account for the impact of the small scales on large-scale physics [64]. Especially, in supersonic flows with high SGS Mach number, the negative values of eddy viscosity are found to be not completely correlated with the occurrence of SGS backscatter due to the increasingly important role of pressure-dilation work [63]. Generally, the interplay between small and large scales of motion become especially complex in the large-eddy simulation of turbulent flows with high compressibility, and thus efforts in developing turbulence models that depict the correct physics should be made at first to remove the barrier in modeling supersonic combustion.

Figure 16 compares the turbulent non-premixed combustion modes in Borghi's diagram for the quarter-domain and full-domain modelings based on a historical statistic of $D a$ versus $R e$. The Damköhler number $D a$ is calculated as the ratio of Taylor time scale $\tau_{t}$ to the chemical time scale $\tau_{c}, D a=\tau_{t} / \tau_{c}$, where $\tau_{t}=\left(k_{\text {res }}+k_{t}\right) / \epsilon, k_{\text {res }}$ is the turbulent kinetic energy of the resolved motions [65], and $\tau_{c}$ is calculated as the same as that used in the PaSR model. The Karlovitz number $K a$ is calculated as $K a=\tau_{c} / \tau_{k}$, where $\tau_{k}=(\nu / \epsilon)^{1 / 2}$ denotes the Kolmogorov time scale. For that $\operatorname{Re} \sim\left(\tau_{t} / \tau_{k}\right)^{2}, R e$ is related to $K a$ and $D a$ by a scaled Reynolds number $R e^{\prime}$ as $R e \sim R e^{\prime}=D a^{2} \cdot K a^{2}$. The current $\tau_{c}$ is defined based on the variation of species across the flame front thickness $l_{F}$, which consists of the thickness of the chemical reaction zone $l_{\delta}$ and the thickness of the preheating zone $l_{T}$. The second Karlovitz number is defined based on the time scale of the chemical reaction zone $\tau_{\delta}$ as $K a_{\delta}=\tau_{\delta} / \tau_{k}$, which is related to $K a$ as $K a_{\delta}=\left(\tau_{\delta} / \tau_{c}\right) K a=\left(l_{\delta} / l_{F}\right)^{2} K a \approx 0.01 K a$ for that $l_{\delta} / l_{F} \approx 0.1$. For non-premixed flames, three combustion modes can be defined based on $K a$ and $D a: 1$ ) flamelet mode with $K a<1$ and $D a>10$; 2) thin reaction zone mode with $1<K a<100$ (equivalent to $\left.K a_{\delta}<1\right)$ and $D a>10 ; 3$ ) slow chemistry mode with $K a>100$ and $D a<10$. Historical statistics show that the percentage of the flamelet mode decreases significantly from 18.2 to $7.42 \%$ when using the full domain, whereas the percentage of the thin reaction zone mode increases from 45.1 to $58.9 \%$. This could be because the reaction processes are closer to the equilibrium in the full-domain modeling; consequently, the chemical time scales increase along with the increase in $K a$ and decrease in $D a$. The percentage of the 


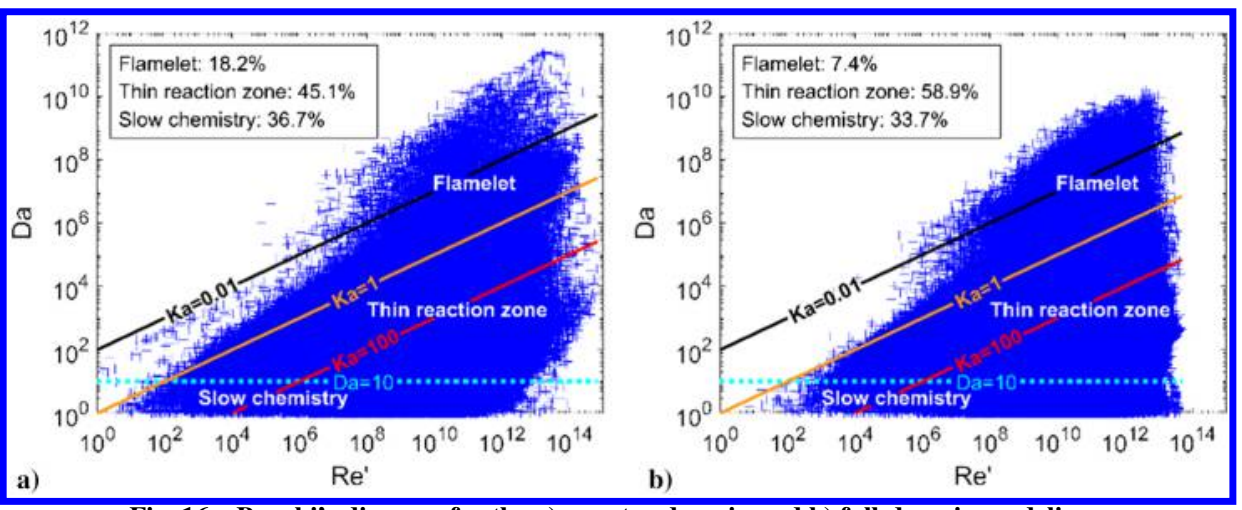

Fig. 16 Borghi's diagram for the a) quarter-domain and b) full-domain modelings.

slow chemistry mode maintains at a high level with only a small variation from 36.7 to $33.7 \%$. Because at least $1 / 3$ of the data points are in the slow chemistry mode, the turbulence-chemistry interaction in kerosene combustion modeling is inaccurate to be handled by combustion models based on fast chemistry assumptions.

\section{Conclusions}

This study quantitatively investigated the influences of symmetry boundary condition, which is a frequently used simplification in modeling symmetric or axisymmetric supersonic combustors. A quarterly split domain and the full domain were modeled using the same physical models and numerical methods, that is, the S-A based IDDES turbulence model, a reformulated PaSR combustion model, a $19 s / 54 r$ skeletal kerosene mechanism, and a third-order SSD discretization scheme. Grid sensitivity analyses with up to 49.20 million cells for the quarter domain and 135.60 million cells for the full domain were conducted.

The mean flow fields were first compared for the quarter-domain and full-domain modelings. For both domains, satisfactory agreements on the mean pressure prediction are achieved at the measuring points. The full-domain modeling predicts a slightly higher peak pressure compared with the quarter-domain modeling and well captures the small initial pressure rise near the flow entry. The hightemperature combustion gas propagates more upstream in the fulldomain modeling. The mean Mach number distribution shows that the most shrunken supersonic core shifts from the downstream cavity to the upstream cavity when the full domain is applied. Both the pyrolysis reactions and the combustion reactions are significantly intensified in the full-domain modeling. A secondary positive peak of the heat release rate indicates that a noticeable ignition occurs immediately after the fuel injection in the full-domain modeling. Statistics on the flow directions across the symmetry planes shows that clockwise swirling is dominant for most of the streamwise locations, indicating the occurrence of a symmetry breaking under the current area-expansion ratio of 1.57. The mean streamlines show that the flow symmetry is better on the minor symmetry plane than on the major symmetry plane. Asymmetric boundary-layer thickening/ separation can be observed in the full-domain modeling.

Influence of species exchanges on the chemical reactions was analyzed. Historical statistics show that the typical temperature and pressure ranges in the reaction zones are 2000-2500 K and 0.5-1.8 bar, and the rich-shifting phenomenon was observed. Reaction path analysis for three typical statuses indicates that the key species in determining the bulk consumption of fuel molecules and the production of $\mathrm{CO}_{2}$ are $\mathrm{C}_{2} \mathrm{H}_{4}, \mathrm{C}_{2} \mathrm{H}_{3}, \mathrm{CH}_{3}, \mathrm{CH}_{2} \mathrm{O}, \mathrm{HCO}$, and $\mathrm{CO}$, which are all among the highest rank of imbalance. The bilateral exchanges of those key species greatly facilitate the autoignition and flame stabilization and thus are responsible for the higher heat release rate in the full-domain modeling. The mass, force, and high-temperature gas have relatively small exchange imbalances compared with the reacting species, indicating that the ensemble flow has statistically symmetric distribution in mass, momentum, and temperature. The mixing efficiencies for the two domains are similar with the final values of 0.89 versus 0.91 , but the combustion efficiency has a rise from 0.74 to 0.95 . The total pressure loss has a drastic rise when using the full domain, but the final losses are almost identical with all approaching 0.8 .

Influences of the symmetry domain on the turbulence and turbulence-chemistry interaction were analyzed. The turbulence spectrums exhibit a $-8 / 3$ power-law behavior for the inertial subrange, which is in consistency with the previous observations for supersonic flows. A shorter inertial subrange is observed for the quarter-domain modeling as the split domain confines the largest eddies. In the quarter-domain modeling, a unique spike structure is observed at the end of the inertial subrange, where the energy is accumulated from the backscatter energy flux from the smaller scales and the forward scatter energy flux from the inertial subrange. Historical statistics in the Borghi's diagram shows that the percentage of the flamelet mode decreases significantly from 18.2 to $7.42 \%$ when using a full domain, whereas the percentage of the thin reaction zone mode increases from 45.1 to $58.9 \%$. The fact that the slow chemistry mode maintains around $1 / 3$ suggests that those fast-chemistry-assumed combustion models are inaccurate to handle the turbulence-chemistry interaction for supersonic kerosene combustion modeling.

\section{Acknowledgments}

The research is supported by Training Program of the Major Research Plan of National Natural Science Foundation of China (Grant No. 91641110), National Natural Science Foundation of China (Grant No. 11502270), and LHD Innovative Foundation (Grant No. LHD2018JS01). The authors are grateful to National Supercomputer Center in Tianjin for providing the computational resource.

\section{References}

[1] Zhang, M., Hu, Z., He, G., and Liu, P., "Large-Eddy Simulation of Kerosene Spray Combustion in a Model Scramjet Chamber," Proceedings of the Institution of Mechanical Engineers, Part G: Journal of Aerospace Engineering, Vol. 224, No. 9, 2010, pp. 949-960. doi:10.1243/09544100JAERO738

-[2] Fureby, C., Chapuis, M., Fedina, E., and Karl, S., "CFD Analysis of the Hyshot Ii Scramjet Combustor," Proceedings of the Combustion Institute, Vol. 33, No. 2, 2011, pp. 2399-2405. doi:10.1016/j.proci.2010.07.055

[3] David, P., Russell, B., and Vincent, W., "Hybrid Reynolds-Averaged and Large-Eddy Simulation of Mixing in an Axisymmetric Scramjet," AIAA Paper 2012-5902, 2012. doi: $10.2514 / 6.2012-5902$

[4] Houshang, E., Datta, G., and Faure, M.-M., "Exploratory RANS and LES Simulations of Transient Supersonic Combustor Flow," AIAA Paper 2009-128, 2009. doi: $10.2514 / 6.2009-128$

[5] Chapuis, M., Fedina, E., Fureby, C., Hannemann, K., Karl, S., and Martinez Schramm, J., "A Computational Study of the Hyshot II Combustor Performance," Proceedings of the Combustion Institute, Vol. 34, No. 2, 2013, pp. 2101-2109. doi:10.1016/j.proci.2012.07.014 
[6] Capra, B. R., Brown, L. M., and Boyce, R. R., "Aerothermal-Structural Analysis of a Rocket-Launched Mach 8 Scramjet Experiment: Descent," Journal of Spacecraft and Rockets, Vol. 52, No. 3, 2015, pp. 684-696. doi:10.2514/1.A33112

[7] Lacaze, G., Vane, Z., and Oefelein, J. C., "Large Eddy Simulation of the Hifire Direct Connect Rig Scramjet Combustor," AIAA Paper 2017-0142, 2017. doi:10.2514/6.2017-0142

[8] Duwig, C., and Fureby, C., "Large Eddy Simulation of Unsteady Lean Stratified Premixed Combustion," Combustion and Flame, Vol. 151, Nos. 1-2, 2007, pp. 85-103. doi:10.1016/j.combustflame.2007.04.004

- [9] Sharpe, G. J., and Falle, S. A. E. G., "Nonlinear Cellular Instabilities of Planar Premixed Flames: Numerical Simulations of the Reactive Navier-Stokes Equations," Combustion Theory and Modelling, Vol. 10, No. 3, 2006, pp. 483-514. doi:10.1080/13647830500472354

- [10] Bayliss, A., and Matkowsky, B. J., "Nonlinear Dynamics of Cellular Flames," SIAM Journal on Applied Mathematics, Vol. 52, No. 2, 1992, pp. 396-415. doi: $10.1137 / 0152022$

[11] Urzay, J., O'Brien, J., Ihme, M., Moin, P., and Saghafia, A., "Backscatter of Turbulent Kinetic Energy in Chemically-Reacting Compressible Flow," Center for Turbulence Research, Annual Research Briefs 2013, Stanford, CA, 2013, pp. 123-139.

[12] O'Brien, J., Urzay, J., Poludnenko, A. Y., Hamlington, P. E., and Ihme, M., "Counter-Gradient Subgrid-Scale Transport and Energy Backscatter in Turbulent Deflagrations," Center for Turbulence Research Proceedings of the 2014 Summer Program, Stanford University, CA, 2014, pp. 147-157.

[13] Staschus, C., and Frederick, R. A., "An Overview of Combustion Instabilities and Rocket Engine Injector Design," AIAA Paper 2016-4724, 2016. doi:10.2514/6.2016-4724

[14] Urzay, J., "Supersonic Combustion in Air-Breathing Propulsion Systems for Hypersonic Flight," Annual Review of Fluid Mechanics, Vol. 50, No. 1, 2018, pp. 593-627. doi:10.1146/annurev-fluid-122316-045217

[15] Gicquel, L. Y. M., Staffelbach, G., and Poinsot, T., "Large Eddy Simulations of Gaseous Flames in Gas Turbine Combustion Chambers," Progress in Energy and Combustion Science, Vol. 38, No. 6, 2012, pp. 782-817. doi:10.1016/j.pecs.2012.04.004

[16] Bauerheim, M., Salas, P., Nicoud, F., and Poinsot, T., "Symmetry Breaking of Azimuthal Thermo-Acoustic Modes in Annular Cavities: A theoretical Study," Journal of Fluid Mechanics, Vol. 760, Nov. 2014, pp. 431-465. doi:10.1017/jfm.2014.578

-117] Shur, M. L., Spalart, P. R., Strelets, M. K., and Travin, A. K., "A Hybrid RANS-LES Approach with Delayed-DES and Wall-Modelled LES Capabilities," International Journal of Heat and Fluid Flow, Vol. 29, No. 6, 2008, pp. 1638-1649. doi:10.1016/j.ijheatfluidflow.2008.07.001

[18] Greenshields, C. J., Weller, H. G., Gasparini, L., and Reese, J. M., "Implementation of Semi-Discrete, Non-Staggered Central Schemes in a Colocated, Polyhedral, Finite Volume Framework, for High-Speed Viscous Flows," International Journal for Numerical Methods in Fluids, Vol. 38, No. 2, 2009, pp. 139-161. doi:10.1002/fld.2069

[19] Weller, H. G., Tabor, G., Jasak, H., and Fureby, C., "A Tensorial Approach to CFD Using Object Oriented Techniques," Computers in Physics, Vol. 12, No. 6, 1998, pp. 620-631. doi:10.1063/1.168744

[20] Kee, R. J., Rupley, F. M., and Miller, J. A., "Chemkin-Ii: A Fortran Chemical Kinetics Package for the Analysis of Gas-Phase Chemical Kinetics," Sandia National Laboratories TR SAND89-8009B, Albuquerque, NM, Sept. 1989.

[21] Huber, M. L., "Nist Thermophysical Properties of Hydrocarbon Mixtures Database (Supertrapp) V3.1," NIST Standard Reference Database 4, National Institute of Standards and Technology, Boulder, CO, Feb. 2003.

[22] Kurganov, A., and Tadmor, E., "New High-Resolution Central Schemes for Nonlinear Conservation Laws and Convection-Diffusion Equations," Journal of Computational Physics, Vol. 160, No. 1, 2000, pp. 241-282 doi:10.1006/jcph.2000.6459

[23] Vuorinen, V., Larmi, M., Schlatter, P., Fuchs, L., and Boersma, B. J., "A Low-Dissipative, Scale-Selective Discretization Scheme for the
Navier-Stokes Equations," Computers \& Fluids, Vol. 70, Nov. 2012, pp. 195-205.

doi:10.1016/j.compfluid.2012.09.022

[24] Crank, J., and Nicotson, P., "A Practical Method for Numerical Evaluation of Solutions of Partial Differential Equations of the HeatConduction Type," Advances in Computational Mathematics, Vol. 6, No. 1, 1996, pp. 207-226.

doi:10.1007/BF02127704

[25] Wu, K., Li, X., Yao, W., and Fan, X., "Three-Dimensional Numerical Study of the Acoustic Properties of a Highly Underexpanded Jet," AIAA Paper 2015-3572, 2015. doi: $10.2514 / 6.2015-3572$

[26] Li, X., Wu, K., Yao, W., and Fan, X., "A Comparative Study of Highly Underexpanded Nitrogen and Hydrogen Jets Using Large Eddy Simulation," AIAA Paper 2015-3573, 2015. doi:10.2514/6.2015-3573

-[27] Li, X., Yao, W., and Fan, X., "Large-Eddy Simulation of Time Evolution and Instability of Highly Underexpanded Sonic Jets," AIAA Journal, Vol. 54, No. 10, 2016, pp. 3191-3211. doi: 10.2514/1.J054689

[28] Li, X., Zhou, R., Yao, W., and Fan, X., "Flow Characteristic of Highly Underexpanded Jets from Various Nozzle Geometries," Applied Thermal Engineering, Vol. 125, Oct. 2017, pp. 240-253. doi:10.1016/j.applthermaleng.2017.07.002

[29] Li, X., Fan, E., Yao, W., and Fan, X., "Numerical Investigation of Characteristic Frequency Excited Highly Underexpanded Jets," Aerospace Science and Technology, Vol. 63, April 2017, pp. 304-316. doi:10.1016/j.ast.2017.01.005

[30] Lee, Y., Yao, W., and Fan, X., "A Low-Dissipation Solver Based on Openfoam Designed for Large Eddy Simulation in Compressible Flows," AIAA Paper 2017-2444, 2017. doi: $10.2514 / 6.2017-2444$

- [31] Li, X., Wu, K., Yao, W., and Fan, X., "A Comparative Study of Highly Underexpanded Nitrogen and Hydrogen Jets Using Large Eddy Simulation," International Journal of Hydrogen Energy, Vol. 41, No. 9 , 2016, pp. 5151-5161. doi:10.1016/j.ijhydene.2016.01.120

[32] Yao, W., Wang, J., Lu, Y., Li, X., and Fan, X., "Full-Scale Detached Eddy Simulation of Kerosene Fueled Scramjet Combustor Based on Skeletal Mechanism," AIAA Paper 2015-3579, 2015. doi: 10.2514/6.2015-3579

[33] Wu, K., Zhang, P., Yao, W., and Fan, X., "Numerical Investigation on Flame Stabilization in DLR Hydrogen Supersonic Combustor with Strut Injection," Combustion Science and Technology, Vol. 189, No. 12, 2017, pp. 2154-2179. doi:10.1080/00102202.2017.1365847

[34] Yao, W., Lu, Y., Li, X., Wang, J., and Fan, X., "Improved Delayed Detached Eddy Simulation of a High-Ma Active-Cooled Scramjet Combustor Based on Skeletal Kerosene Mechanism," AIAA Paper 2016-4761, 2016.

doi:10.2514/6.2016-476

[35] Yao, W., Yuan, Y., Li, X., Wang, J., Wu, K., and Fan, X., "Comparative Study of Elliptic and Round Scramjet Combustors Fueled by Rp-3," Journal of Propulsion and Power, Vol. 34, No. 3, 2018, pp. 772-786. doi:10.2514/1.B36721

[36] Wu, K., Yao, W., and Fan, X., "Development and Fidelity Evaluation of a Skeletal Ethylene Mechanism Under Scramjet-Relevant Conditions," Energy and Fuels, Vol. 31, No. 12, 2017, pp. 14296-14305. doi:10.1021/acs.energyfuels.7b03033

[37] Yao, W., Lu, Y., Wu, K., Wang, J., and Fan, X., "Modeling Analysis of an Actively-Cooled Scramjet Combustor Under Different Kerosene/Air Ratios," Journal of Propulsion and Power, Vol. 34, No. 4, 2018, pp. $975-991$. doi:10.2514/1.B36866

[38] Bird, R. B., Stewart, W. E., and Lightfoot, E. N., "Viscosity and the Mechanisms of Momentum Transport," Transport Phenomena, 2nd ed., Wiley, New York, 2002, p. 27.

[39] Spalart, P. R., and Allmaras, S. R., "A One-Equation Turbulence Model for Aerodynamic Flows," AIAA Paper 1992-0439, 1992. doi:10.2514/6.1992-439

[40] Golovitchev, V. I., Nordin, N., Jarnicki, R., and Chomiak, J., "3-D Diesel Spray Simulations Using a New Detailed Chemistry Turbulent Combustion Model," SAE TP 2000-01-18912000. doi:10.4271/2000-01-1891

[41] Pope, S. B., "Computationally Efficient Implementation of Combustion Chemistry Using in Situ Adaptive Tabulation," Combustion Theory and Modeling, Vol. 1, No. 1, 1997, pp. 41-63. doi: $10.1080 / 713665229$ 
[42] Fureby, C., "LES for Supersonic Combustion," AIAA Paper 2012-5979, 2012. doi:10.2514/6.2012-5979

[43] Dagaut, P., Karsenty, F., Dayma, G., Diévart, P., Hadj-Ali, K., MzéAhmed, A., Braun-Unkhoff, M., Herzler, J., Kathrotia, T., Kick, T., et al., "Experimental and Detailed Kinetic Model for the Oxidation of a Gas to Liquid (GtL) Jet Fuel," Combustion and Flame, Vol. 161, No. 3, 2014, pp. 835-847. doi:10.1016/j.combustflame.2013.08.015

[44] Niemeyer, K. E., Sung, C.-J., and Raju, M. P., "Skeletal Mechanism Generation for Surrogate Fuels Using Directed Relation Graph with Error Propagation and Sensitivity Analysis," Combustion and Flame, Vol. 157, No. 9, 2010, pp. 1760-1770. doi:10.1016/j.combustflame.2009.12.022

[45] Clarke, D. K., Hassan, H. A., and Salas, M. D., "Euler Calculations for Multielement Airfoils Using Cartesian Grids," AIAA Journal, Vol. 24, No. 3, 1986, pp. 353-358. doi: $10.2514 / 3.9273$

[46] Fan, X., and Yu, G., "Analysis of Thermophysical Properties of Daqing Rp-3 Aviation Kerosene," Journal of Propulsion Technology, Vol. 27, No. 2, 2006, pp. 187-192.

[47] Lu, Y., Wang, X., Li, L., Cheng, D., Yao, W., and Fan, X., "Development and Preliminary Validation of a Thermal Analysis Method for Hydrocarbon Regenerative-Cooled Supersonic Combustor," AIAA Paper 2015-3556, 2015. doi:10.2514/6.2015-3556

[48] Abbott, D. E., and Kline, S. J., "Experimental Investigation of Subsonic Turbulent Flow over Single and Duble Backward Facing Steps," Journal of Basic Engineering, Vol. 84, No. 3, 1962, pp. 317-325. doi: $10.1115 / 1.3657313$

[49] Law, C. K., Makino, A., and Lu, T. F., "On the Off-Stoichiometric Peaking of Adiabatic Flame Temperature," Combustion and Flame, Vol. 145, No. 4, 2006, pp. 808-819. doi:10.1016/j.combustflame.2006.01.009

[50] Sun, W., Chen, Z., Gou, X., and Ju, Y., "A Path Flux Analysis Method for the Reduction of Detailed Chemical Kinetic Mechanisms," Combustion and Flame, Vol. 157, No. 7, 2010, pp. 1298-1307. doi:10.1016/j.combustflame.2010.03.006

[51] McGuire, J. R., Boyce, R. R., and Mudford, N. R., "Radical-Farm Ignition Processes in Two-Dimensional Supersonic Combustion," Journal of Propulsion and Power, Vol. 24, No. 6, 2008, pp. 1248-1257. doi:10.2514/1.35562

[52] Rajasekaran, A., and Babu, V., "Numerical Simulation of ThreeDimensional Reacting Flow in a Model Supersonic Combustor," Journal of Propulsion and Power, Vol. 22, No. 4, 2006, pp. 820-827. doi:10.2514/1.14952

[53] Bilger, R. W., Starner, S. H., and Kee, R. J., "On Reduced Mechanisms for Methane-Air Combustion in Non-Premixed Flames," Combustion and Flame, Vol. 80, No. 2, 1990, pp. 135-149. doi:10.1016/0010-2180(90)90122-8
[54] You, Y., Ludeke, H., and Hannemann, K., "On the Flow Physics of a Low Momentum Flux Ratio Jet in a Supersonic Turbulent Crossflow," Europhysics Letters, Vol. 97, No. 2, 2012, Paper 24001. doi:10.1209/0295-5075/97/24001

[55] Clercx, H. J., and Van Heijst, G. J., "Energy Spectra for Decaying 2D Turbulence in a Bounded Domain," Physical Review Letters, Vol. 85, No. 2, 2000, pp. 306-309. doi:10.1103/PhysRevLett.85.306

[56] Ingenito, A., and Bruno, C., "Physics and Regimes of Supersonic Combustion," AIAA Journal, Vol. 48, No. 3, 2010, pp. 515-525. doi:10.2514/1.43652

[57] Ingenito, A., Cecere, D., and Giacomazzi, E., "Large Eddy Simulation of Turbulent Hydrogen-Fuelled Supersonic Combustion in an Air Cross-Flow," Shock Waves, Vol. 23, No. 5, 2013, pp. 481-494. doi:10.1007/s00193-013-0454-7

[58] Kritsuk, A. G., Padoan, P., Wagner, R., and Norman, M. L., "Scaling Laws and Intermittency in Highly Compressible Turbulence," AIP Conference Proceedings, AIP, Vol. 932, No. 1, 2007, pp. 393-399. doi:10.1063/1.2778991

[59] Kritsuk, A. G., Norman, M. L., Padoan, P., and Wagner, R., "The Statistics of Supersonic Isothermal Turbulence," The Astrophysical Journal, Vol. 665, No. 1, 2007, pp. 416-431. doi:10.1086/509297

[60] Biagioni, L., and d'Agostino, L., "Measurement of Energy Spectra in Weakly Compressible Turbulence," AIAA Paper 1999-3516, 1999. doi:10.2514/6.1999-3516

[61] Elsinga, G. E., and Marusic, I., "The Anisotropic Structure of Turbulence and Its Energy Spectrum," Physics of Fluids, Vol. 28, No. 1, 2016, Paper 011701. doi:10.1063/1.4939471

[62] Yue, W., Meneveau, C., Parlange, M. B., Zhu, W., Kang, H. S., and Katz, J., "Turbulent Kinetic Energy Budgets in a Model Canopy: Comparisons Between LES and Wind-Tunnel Experiments," Environmental Fluid Mechanics, Vol. 8, No. 1, 2008, pp. 73-95. doi:10.1007/s10652-007-9049-0

[63] O’Brien, J., Urzay, J., Ihme, M., Moin, P., and Saghafian, A., "SubgridScale Backscatter in Reacting and Inert Supersonic Hydrogen-Air Turbulent Mixing Layers," Journal of Fluid Mechanics, Vol. 743, March 2014, pp. 554-584. doi:10.1017/jfm.2014.62

[64] Meneveau, C., and Katz, J., "Scale-Invariance and Turbulence Models for Large-Eddy Simulation," Annual Review of Fluid Mechanics, Vol. 32, No. 1, 2000, pp. 1-32. doi:10.1146/annurev.fluid.32.1.1

[65] Pope, S. B., "Ten Questions Concerning the Large-Eddy Simulation of Turbulent Flows," New Journal of Physics, Vol. 6, March 2004, p. 35. doi: $10.1088 / 1367-2630 / 6 / 1 / 035$

J. C. Oefelein Associate Editor 
This article has been cited by:

1. Bo Li, Wei Yao, Yachao Lee, XueJun Fan. 2021. Reconstruction model for heat release rate based on artificial neural network. International Journal of Hydrogen Energy 46:37, 19599-19616. [Crossref]

2. Wei Yao, Liu Chen. Large Eddy Simulation of REST Hypersonic Combustor based on Dynamic Zone Flamelet Model . [Abstract] [PDF] [PDF Plus]

3. Wei Yao. 2020. On the application of dynamic zone flamelet model to large eddy simulation of supersonic hydrogen flame. International Journal of Hydrogen Energy 45:41, 21940-21955. [Crossref]

4. Wei Yao. 2019. Kerosene-fueled supersonic combustion modeling based on skeletal mechanisms. Acta Mechanica Sinica 35:6, 1155-1177. [Crossref]

5. Wei Yao, Yichao Jin, Yachao Li. Stiff phase-change phenomenon modeled by a compressible LES solver . [Citation] [PDF] [PDF Plus]

6. Wei Yao, Xuejun Fan. Application of dynamic zone flamelet model to a GH2/GO2 rocket combustor . [Citation] [PDF] [PDF Plus] 